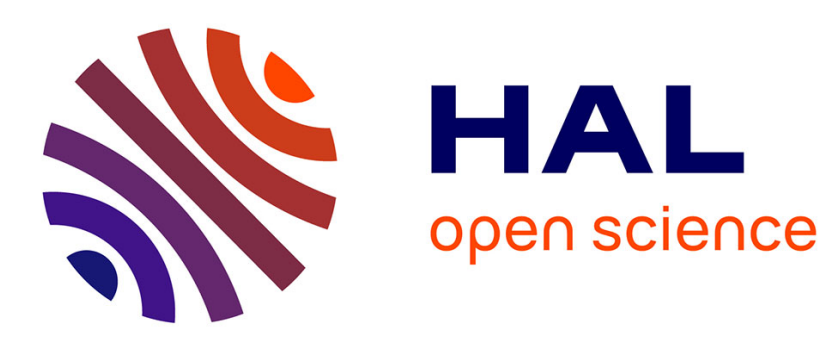

\title{
Solid-fluid phase transformation within grain boundaries during compaction by pressure solution
}

Joumana Ghoussoub, Yves Leroy

\section{To cite this version:}

Joumana Ghoussoub, Yves Leroy. Solid-fluid phase transformation within grain boundaries during compaction by pressure solution. Journal of the Mechanics and Physics of Solids, 2001, 49 (10), pp.2385-2430. 10.1016/S0022-5096(01)00012-6 . hal-00111330

\section{HAL Id: hal-00111330 \\ https://hal.science/hal-00111330}

Submitted on 26 Sep 2019

HAL is a multi-disciplinary open access archive for the deposit and dissemination of scientific research documents, whether they are published or not. The documents may come from teaching and research institutions in France or abroad, or from public or private research centers.
L'archive ouverte pluridisciplinaire HAL, est destinée au dépôt et à la diffusion de documents scientifiques de niveau recherche, publiés ou non, émanant des établissements d'enseignement et de recherche français ou étrangers, des laboratoires publics ou privés. 


\title{
Solid-fluid phase transformation within grain boundaries during compaction by pressure solution
}

\author{
Joumana Ghoussoub, Yves M. Leroy* \\ Laboratoire de Mécanique des Solides, École polytechnique, U.M.R. C.N.R.S. no. 7649, \\ 91128 Palaiseau Cedex, France
}

\begin{abstract}
The overall compaction of porous rocks due to intergranular pressure solution (IPS) results from the dissolution of minerals within contact regions and the diffusive transport through the grain boundary of the dissolved species towards the fluid-filled pore space. The grain boundary structure can be imagined to be composed of dry contact zones, thin fluid films and fluid-filled cavities. The connectiveness and tortuosity of this structure determine the effective diffusivity of grain contacts and thus the potential of porous rock to compact by the action of IPS. The evolution in time of the grain-boundary structure, and thus of the effective diffusivity, is discussed here with the help of two 2D initial- and boundary-value problems which are solved by analytical and numerical means. The evolution of the solid-fluid interfaces within the grain boundary is governed by a phase transformation between the non-hydrostatically stressed elastic solid and the trapped fluid assumed in mechanical equilibrium. The characteristic time is provided by a linear kinetic law. The evolution of the structure away from a state of thermodynamic equilibrium during a loading normal to the grain boundary is found to occur in two steps. The first one consists of a diffuse morphology evolution in time and results in an enhancement of any initial stress concentration. The second step is characterized by a rapid and localized dissolution in the region of stress concentration. The latency period prior to localization is governed by the magnitude of the non-hydrostatic remote stress as well as the microstructural geometric factor responsible for the initial stress concentration at the solid-fluid interface. The localized dissolution is shown to provide a mechanism for the fluid to penetrate a previously dry contact region by marginal dissolution and thus to create a fluid film. However, the newly formed thin fluid layer is found to be unstable pointing to a possible repeated reorganization or dynamic evolution of the grain boundary internal structure during the action of IPS.
\end{abstract}

Keywords: A. Grain boundaries; A. Phase transformation; B. Geological material; B. Finite elements; C. Stability

\footnotetext{
* Corresponding author. Tel.: +33-16933-3358; fax: +33-16933-3026.
}

E-mail address: leroyy@lms.polytechnique.fr (Y. M. Leroy). 


\section{Introduction}

The objective of this paper is to study the evolution with time of the grain boundary internal structure under conditions typical of the action of intergranular pressure solution (IPS).

The deformation mechanism IPS differs from classical Coble creep mainly by the presence of a fluid phase within the grain boundary. IPS consists of the dissolution of minerals at stressed contact points, transport of the dissolved material through the grain boundary and its redeposition elsewhere. It occurs in the Earth's crust at geological rates and results in lithification and compaction of low grade metamorphic rocks (Rutter, 1983). IPS is partly responsible for the reduction in porosity of sedimentary rocks, a key parameter in determining the quality of potential hydrocarbon reservoirs. IPS has also been invoked to explain the creeping and the strength recovery of faults (Sleep, 1995). Finally, it is active in halite (Urai et al., 1986), a material which has been studied extensively for the safety of nuclear and industrial waste disposals.

Rheological models for IPS often include the assumption that the grain boundary is a Gibbs interface having some effective diffusivity responsible for the transport of dissolved minerals (Rutter, 1976; Lehner and Bataille, 1984/85; Spiers and Schutjens, 1990; Paterson, 1995). The diffusivity coefficient is a phenomenological constant and is deduced from experiments performed on a collection of compacting grains. This choice of a constant value cannot model the exact nature of the action of IPS at the grain boundary scale and the potential healing of grain boundaries which would prevent the action of IPS. Three models have been proposed to describe the action of IPS at the grain boundary scale. The first one assumes that the dissolution proceeds from the pore space towards the inner contact region (Bathurst, 1958) and could be coupled with some crystal plasticity. The key feature of the second model is the existence of an adsorbed fluid film at the grain boundary (Weyl, 1959). The third model assumes the existence of a network of islands and channels which evolves with time and whose tortuosity and connectiveness result in an effective grain boundary diffusivity (Raj, 1982).

Evidence on the nature of the grain boundary structure during the action of pressure solution is difficult to obtain in the laboratory. One of the reasons is, of course, the characteristic time of the process which has to be shortened in the laboratory by changing the chemistry of the fluid phase (Gratier and Guiguet, 1986) or by increasing the temperature. For instance, Cox and Paterson (1991) have been able to show through an experiment on a fine powder of quartz grains at $1200 \mathrm{~K}$ that there was indeed a network of islands and channels within the grain contact. Similar results have been obtained at room temperature on rock salt, which should be seen as an analogue material, by Urai et al. (1986) and Spiers et al. (1990). These conclusions have been challenged by Hickman and Evans (1995) who advocated, from their observations of contacts between polished lenses of halite, that no fluid phase could be maintained within a contact to allow IPS to occur.

The question of the internal structure of the grain boundary will be solved only by observations collected during the action of IPS. This paper proposes, in parallel to 
the ongoing experimental effort (Schutjens and Spiers, 1999), a numerical modeling of the evolution with time of the grain boundary structure. The solution of initialand boundary-value problems should thus become the mere consequence of accepted assumptions on IPS, which are discussed next, and is used to shed light on the nature of the deformation mechanism.

The first basic and well-accepted assumption to model IPS is that the solid-fluid interaction can be described as a phase transformation between a non-hydrostatically stressed solid and a fluid. In addition, the solid is considered here to be isotropic and elastic and the fluid in mechanical and chemical equilibrium. The fluid phase is composed of a diluent (e.g., water) and a single solute of the same composition as the solid phase. The characteristic length of the problem is less than the grain boundary thickness, so that the diffusion time is short compared to the kinetics of the phase transformation. The kinetic law was derived using concepts of irreversible thermodynamics by Lehner and Bataille (1984/85) and then extended by Heidug and Leroy (1994) to include surface tension effects. The kinetic driving force is, of course, zero if Gibbs' (1878) equilibrium condition is met.

Little work has been reported so far in the literature on IPS as an initial- and boundary-value problem. The few contributions are concerned with the initial morphology change in time of a solid-fluid interface using linear stability arguments. For example, Leroy and Heidug (1994) found that the agent driving the instability of a planar surface is the jump in tangential stress component across the solid-fluid interface. Surface tension stabilizes the shorter wavelength modes and provides the characteristic length responsible for the selection of a dominant mode of instability. These findings have many similarities with known results on the stability of interfaces driven by surface diffusion (Srolovitz, 1989), which has its roots in the seminal contribution of Mullins and Sekerka (1963). Stability analyses which differ by the choice of relevant characteristic time should, of course, provide the same neutral stability predictions if they assume the same conditions for thermodynamic equilibrium (Grinfeld, 1991). Non-linear evolution with time of a solid-fluid interface, which is the main objective of this paper, has received less attention apart from the effort on solidification and melting processes (e.g., Zabaras et al., 1991). At the scale of the grain boundary, non-linear evolution due to surface diffusion has been considered by Chuang et al. (1979) to capture the relation between time for cavitation and both defect geometry and applied stress. Detailed calculations with Green's functions have revealed the formation of a cusp from planar surfaces or cylindrical cavities (Chiu and Gao, 1993; Yang and Srolovitz, 1994; Wang and Suo, 1997).

In the next section, one states the governing equations and their dimensional form and expresses the weak formulation for initial- and boundary-value problems. The driving force for phase transformation is presented once general thermodynamic equilibrium conditions are stated in terms of chemical potential tensors (Bowen, 1967). The finite-element method is presented next. Its feature is an enhancement of the continuity of the field variables and the geometrical interpolation on the interface. Further information on the element and its convergence properties are found in Appendix B. The solutions of two initial- and boundary-value problems are presented in Section 4 to shed light on the evolution with time of the grain boundary internal structure. The 
numerical scheme is validated by comparing the results with the analytical predictions of a linear stability analysis, found in Appendix C.

\section{Governing equations, dimensional analysis and weak formulation}

This section pertains first to the presentation of the physics governing the migration of an interface separating a non-hydrostatically stressed solid and a fluid phase in mechanical and chemical equilibrium. The migration results from a phase transformation between a linear elastic, isotropic solid and a fluid containing the same material in solution with a diluent. The kinetics of the phase transformation provides the dominant characteristic time of the interface migration which is thus assumed to be large compared to the diffusion characteristic time within the fluid phase. No diffusion occurs within the solid phase. The physics necessary to describe IPS being introduced, a dimensional analysis is proposed prior to the discussion of the weak formulation for initial- and boundary-value problems.

\subsection{Field equations and kinetic law}

The first objective is to introduce the thermodynamic force responsible for the migration of the solid-fluid interface starting from the general thermodynamic equilibrium condition for two phases composed of several constituents. It is shown how this condition includes Gibbs' (1878) equilibrium for the particular solid-fluid system of interest. This derivation is found useful to cast our analysis in a general framework and also serves the purpose of defining notation.

To define the conditions for thermodynamic equilibrium across an interface, it is convenient to introduce the concept of chemical potential tensor. Bowen (1967) was apparently the first to do so in an attempt to derive a general theory of mixtures without postulating the concept of partial fluxes. The definition of Bowen and Wiese (1969) is amended to express the chemical potential tensor as

$$
\mathbf{K}_{a}=\psi_{a} \rrbracket-\boldsymbol{\sigma}_{a} \bar{\rho}_{a}^{-1}
$$

in terms of $\psi_{a}, \bar{\rho}_{a}$ and $\boldsymbol{\sigma}_{a}$ which are the partial specific Helmholtz free energy, the partial mass density and the partial Cauchy stress acting on the ath constituent in a given phase, respectively. Bold letters are reserved for tensorial quantities. The tensor 『 found in Eq. (1) is the second-order identity tensor. The general condition for thermodynamic equilibrium for every constituent present in two phases separated by an interface $\partial \Omega_{\mathrm{I}}$, which is discussed by Truskinovsky (1984), Heidug and Lehner (1985) and Grinfeld (1991), is written here by stating that the jump in normal component of the vector $\mathbf{K}_{a} \cdot \mathbf{n}$ across that interface must be zero

$$
\mathbf{n} \cdot\left(\llbracket \mathbf{K}_{a} \rrbracket \cdot \mathbf{n}\right)=0 .
$$

The bracket stands for the difference between the function in argument estimated in the two phases across the interface. The vector $\mathbf{n}$ is the normal to the stressed interface and its orientation, required in the following, is towards the fluid phase. 
The definition and the name adopted in Eq. (1) are now justified by looking at the particular case of a fluid phase. The Helmholtz free energy of a fluid phase $\psi_{\mathrm{f}}$ is the sum of the partial Helmholtz free energy weighted by the concentration $C_{a}$ of each constituent. The total stress acting locally is the sum of the partial stresses. For a fluid in mechanical equilibrium, that partial stress state is hydrostatic and equal to the opposite of the pore fluid pressure $p_{\mathrm{f}}$ times the ath species concentration $C_{a}$. In this instance, the chemical potential $\mathbf{K}_{a}$ is isotropic and proportional to $\psi_{a}+C_{a} p_{\mathrm{f}} / \bar{\rho}_{a}$. This scalar is the partial Gibbs free energy of the ath constituent if work can only be performed by volume change. Note that the partial mass density $\bar{\rho}_{a}$ is nothing but $C_{a} \rho_{\mathrm{f}}$ in which $\rho_{\mathrm{f}}$ is the mass density of the fluid phase. Furthermore, by virtue of Euler's theorem, the partial Gibbs free energy equals the chemical potential of the constituent denoted by $\mu_{a}$ and $\mathbf{K}_{a}$ is nothing but $\mu_{a} \rrbracket$. These remarks and definitions, classical in mixture theories (Bowen and Wiese, 1969), are consistent with Gibbs-Duhem equation in the fluid phase:

$$
\mathrm{f}+\frac{p_{\mathrm{f}}}{\rho_{\mathrm{f}}} \equiv \sum_{a} C_{a}\left(\psi_{a}+\frac{p_{\mathrm{f}}}{\rho_{\mathrm{f}}}\right)=\sum_{a} C_{a} \mu_{a},
$$

and justify the origin of the name attributed to the tensor $\mathbf{K}_{a}$ in Eq. (1).

We now specialize the equilibrium condition (2) to the particular solid-fluid system of interest. The fluid phase is composed of two constituents or species, the solute and the diluent. Quantities attached to the former and the latter species are marked by a $S$ and a $D$ in subscript, respectively. The solid phase is composed of a single constituent of the same nature as the solute in the fluid phase. Field quantities assigned to the solid phase are designated by a $s$ in subscript. The solid-fluid interface is envisioned, following the proposition of Gibbs, as a dividing surface of virtual thickness appropriately positioned in the region of rapid variation in densities so that the thermodynamic force associated to the curvature vanishes (Gibbs, 1878). This interface could also be seen, however, as a membrane having specific properties and sustaining its own state of stress and deformation (Alexander and Johnson, 1985; Heidug, 1991; Heidug and Leroy, 1994) to characterize the energy stored in that region. For the present investigation, the simplifying assumption of an isotropic interface is proposed permitting the mechanical equilibrium conditions across the interface to be written as

$$
\begin{aligned}
& \mathbf{t}_{\alpha} \cdot \boldsymbol{\sigma}_{\mathrm{s}} \cdot \mathbf{n}=0, \\
& \mathbf{n} \cdot \boldsymbol{\sigma}_{\mathrm{s}} \cdot \mathbf{n}=2 H \gamma-p_{\mathrm{f}},
\end{aligned}
$$

in terms of the mean curvature $2 H$, surface tension $\gamma$ and two arbitrary vectors $\mathbf{t}_{\alpha}$ tangent to the interface. Eqs. (4) are the classical Laplace conditions which rely on the assumptions that the fluid is in equilibrium $\left(\boldsymbol{\sigma}_{\mathrm{f}}=-p_{\mathrm{f}} \llbracket\right)$ and thus disregard any shear effect along the interface due, for example, to the fluid viscosity.

Combining Eqs. (1) and (4) and the definition provided above for the solute chemical potential with the jump condition (2) provides

$$
\psi_{\mathrm{s}}+\frac{p_{\mathrm{f}}-2 H \gamma}{\rho_{\mathrm{s}}}-\mu_{\mathrm{S}}=0
$$


which is Gibbs' famous thermodynamic equilibrium condition (Gibbs, 1878) for the solute in contact with the solid phase.

Having defined the concept of thermodynamic equilibrium, we now turn our attention to the class of kinetic laws which govern the migration of the solid-fluid interface under conditions which remain close to the equilibrium condition (5) to render applicable the results of irreversible thermodynamics. This discussion is restricted to our particular solid-fluid problem. The solid phase occupies at time $t$ the domain $\Omega(t)$ called the current configuration. If the solid phase is released from any external loading, its position then defines the reference configuration denoted $\Omega_{0}(t)$. A point $\mathbf{X}$ in the domain $\Omega_{0}(t)$ is mapped to $\mathbf{x}$ in $\Omega(t)$ by a finite transformation. Note that dissolution and precipitation change the total mass contained in $\Omega_{0}(t)$ which is thus also time dependent. This change in mass results from the mass flux $J_{n}$ through the solid-fluid interface $\partial \Omega_{\mathrm{I}}(t)$ and is defined per unit area of the interface in its current configuration by

$$
J_{n}=\rho_{\mathrm{s}}\left(\dot{\mathbf{x}} \cdot \mathbf{n}-S_{n}\right),
$$

in terms of $S_{n}$ and $\dot{\mathbf{x}}$ which are, respectively, the speed of displacement of the solidfluid interface and the material velocity of the point $\mathbf{x}$ currently on the interface. It is more convenient in our analysis to employ the mass flux $J_{N}$ per unit area of the interface in the reference configuration. To relate the two fluxes, let one consider a patch on this interface of areas $\mathrm{d} S$ and $\mathrm{d} S_{0}$ and oriented by the normals $n$ and $N$ over the current and reference configurations, respectively, and take note that

$$
J_{n} \mathrm{~d} S=J_{N} \mathrm{~d} S_{0} \equiv-\rho_{0 \mathrm{~s}} S_{N} \mathrm{~d} S_{0} .
$$

The definition of $J_{N}$ relies on the Lagrangian speed of propagation $S_{N}$ and the reference value of the solid density $\rho_{0 \text { s }}$ (Truesdell, 1977, Chapter II.6). According to Eq. (7), the flux within the fluid phase is positive if the non-material velocity $S_{N}$ is negative signalling a dissolution process. A precipitation requires a positive velocity $S_{N}$ and corresponds to an interface migrating towards the fluid phase with a negative flux $J_{N}$. The thermodynamic driving force conjugate to the mass flux $J_{n}$ denoted by $\chi$ is shown by Lehner and Bataille (1984/85) and Heidug and Leroy (1994), from an estimate of the dissipation during migration, to be the left-hand side of Eq. (5), which is the expression taken by the jump condition (2) for the solute and the solid phase in our problem. Any function $\mathscr{L}(\chi)$ having the following property:

$$
J_{n}=\mathscr{L}(\chi) \quad \text { with } \mathscr{L}(\chi) \chi \geq 0 \quad \text { and } \quad \chi=\psi_{\mathrm{s}}+\frac{p_{\mathrm{f}}-2 H \gamma}{\rho_{\mathrm{s}}}-\mu_{\mathrm{S}}
$$

is a candidate for a kinetic law.

To determine the non-material velocity, or speed of propagation, $S_{N}$ of the interface with Eqs. (8) and (7) one needs to know the solute specific chemical potential. It is a function of the pore fluid pressure and the solute concentration. In Ghoussoub (2000), this function is derived for a mixture of ideal solutions and the final expression reads

$$
\mu_{\mathrm{S}}\left(p_{\mathrm{f}}, C_{\mathrm{S}}\right)=\mu_{\mathrm{S}}^{*}+\frac{p_{\mathrm{f}}-p_{\mathrm{f}}^{0}}{\rho_{\mathrm{S}}}+\frac{R T}{M_{\mathrm{S}}} \log \left(\frac{C_{\mathrm{S}}}{C_{\mathrm{S}}^{0}}\right),
$$


in which $\mu_{\mathrm{S}}^{*}$ should be seen as a reference chemical potential taken at a pore fluid pressure $p_{\mathrm{f}}^{0}$ and a solute concentration $C_{\mathrm{S}}^{0}$. The constants $R, T$ and $M_{\mathrm{S}}$ are the universal gas constant, the temperature and the solute molar mass. The problems treated in what follows are partitioned into two classes. The first class includes open systems: the fluid phase exchanges mass with a remote reservoir of infinite dimension so that the fluid pressure and the solute concentration are unaffected by local changes in morphology of the solid-fluid interface. These two quantities are constant during the whole analysis as well as, consequently, the solute chemical potential according to Eq. (9). In the second class of problems, the systems are closed: the fluid phase is trapped within the grain boundary. In such closed systems, the diluent mass is preserved and the pressure and the solute concentration vary with the local evolution of the solid-fluid interface. In the course of this evolution the current volume of the fluid phase $V_{\mathrm{f}}$ and the mass of the solute $m_{\mathrm{S}}$ vary. It involves solute diffusion within an isolated cavity at a rate sufficiently fast for the fluid phase to be still considered homogeneous at all times. Assuming an ideal solution and a constant isothermal compressibility $\kappa_{\mathrm{D}}$ for the diluent, it is shown in Appendix A that the volume of the fluid phase is the following function of the solute mass and fluid pressure:

$$
V_{\mathrm{f}}\left(p_{\mathrm{f}}, m_{\mathrm{S}}\right)=\frac{m_{\mathrm{S}}}{p_{\mathrm{f}}} \frac{p_{\mathrm{f}}^{0} V_{\mathrm{S}}^{0}}{m_{\mathrm{S}}^{0}}+V_{\mathrm{D}}^{0} \exp \left(-\left(p_{\mathrm{f}}-p_{\mathrm{f}}^{0}\right) \kappa_{\mathrm{D}}\right),
$$

in which the superscript 0 identifies the reference state and $V_{\mathrm{D}}$ denotes the volume of the diluent. In our analysis, the volume of the fluid phase as well as the solute mass are known and Eq. (10) provides the means to compute the pore fluid pressure. The pore pressure and the solute concentration $\left(C_{\mathrm{S}}=m_{\mathrm{S}} /\left(m_{\mathrm{S}}+m_{\mathrm{D}}^{0}\right)\right)$ determine the solute chemical potential (9) which is required to compute the speed of propagation pointwise along the solid-fluid interface.

\subsection{Further assumptions and dimensional analysis}

The objective of this second subsection is to provide the governing equations in a dimensionless form with the assumption of infinitesimal transformation (infinitesimal strain and displacement). While doing so, further assumptions are provided to complete the definition of our problem.

The solid phase is assumed to be free of stress in its reference configuration and to be homogeneous, isotropic and linear elastic. Any new material deposited on the solid phase from the fluid phase is assumed to be added coherently (no build up of residual stresses) and to have the same elastic properties as the material initially in a solid state. The elastic properties of the solid phase are thus described by $\tilde{G}$ and $\tilde{K}$, the modulus of elasticity in shear and bulk deformation, respectively. A superposed tilde over any constant or field variable signals that this physical quantity has dimension. This convention was, of course, not adopted in the previous part of the section. Every dimensionless variable of generic name $A(\mathbf{x}, t)$ is obtained by dividing the physical quantity $\tilde{A}(\mathbf{x}, t)$ by the reference value $A_{\mathrm{R}}$. Reference values will henceforth be denoted by a subscript letter $\mathrm{R}$. These definitions, which are summarized in Table 1, are now discussed. 
Table 1

Characteristic or reference values for physical quantities required for the dimensional analysis, assuming a linear kinetic law as in Lehner and Bataille (1984/85) for a silica-water reaction at a temperature of $217^{\circ} \mathrm{C}$ (Rimstidt and Barnes, 1980)

\begin{tabular}{|c|c|c|c|c|}
\hline Physical quantity & $\begin{array}{l}\text { Reference } \\
\text { quantity }\end{array}$ & Definition & Value & Unit \\
\hline Stress & $\sigma_{\mathrm{R}}$ & Lithostatic pressure at $1 \mathrm{~km}$ depth & $10^{7}$ & $\mathrm{~Pa}$ \\
\hline Surface tension & $\gamma_{\mathrm{R}}$ & For solid-fluid interface & 0.1 & $\mathrm{~Pa} \mathrm{~m}$ \\
\hline Length & $L_{\mathrm{R}}$ & Defined as $\gamma_{R} / \sigma_{R}$ & $10^{-8}$ & $\mathrm{~m}$ \\
\hline Mass density & $\rho_{\mathrm{R}}$ & Mass density of quartz & 2650 & $\mathrm{~kg} / \mathrm{m}^{3}$ \\
\hline Driving force & $\mathscr{X}_{\mathrm{R}}$ & Defined as $\sigma_{\mathrm{R}} / \rho_{\mathrm{R}}$ & 3774 & $\mathrm{~Pa} \mathrm{~m}^{3} / \mathrm{kg}$ \\
\hline Chemical potential & $\mu_{\mathrm{R}}$ & Same as $\mathscr{X}_{\mathrm{R}}$ & 3774 & $\mathrm{~Pa} \mathrm{~m}^{3} / \mathrm{kg}$ \\
\hline Time & $\begin{array}{l}t_{\mathrm{R}}=\rho_{\mathrm{R}}^{2} L_{\mathrm{R}} \\
/\left(\mathscr{L}_{\mathrm{R}} \sigma_{\mathrm{R}}\right)\end{array}$ & $\begin{array}{l}\text { Time lapse to migrate the interface } \\
\text { over } L_{\mathrm{R}} \text { under a driving force } \mathscr{X}_{\mathrm{R}}\end{array}$ & $6.4 \times 10^{5}$ & $\mathrm{~s}$ \\
\hline Mass flux & $J_{\mathrm{R}}$ & Defined as $\rho_{\mathrm{R}} L_{\mathrm{R}} / t_{\mathrm{R}}$ & 414 & $\mathrm{~kg} /\left(\mathrm{m}^{2} \mathrm{~s}\right)$ \\
\hline Kinetics constant & $\mathscr{L}_{\mathrm{R}}$ & Defined as $\rho_{\mathrm{R}}^{2} L_{\mathrm{R}} /\left(t_{\mathrm{R}} \sigma_{\mathrm{R}}\right)$ & $1.1 \times 10^{-14}$ & $\mathrm{~kg} \mathrm{~s} / \mathrm{m}^{4}$ \\
\hline
\end{tabular}

The mass density reference value $\rho_{\mathrm{R}}$ is set to the solid mass density in its reference configuration $\rho_{0 \mathrm{~s}}$ for the particular case of quartz. Any stress $\tilde{\boldsymbol{\sigma}}$ is normalized by the characteristic stress $\sigma_{\mathrm{R}}$ which is chosen as the magnitude of the lithostatic pressure in the Earth's crust at $1 \mathrm{~km}$ depth. Surface tension $\tilde{\gamma}$ has dimension of stress times length and the reference $\gamma_{R}$, used by Leroy and Heidug (1994), is 0.1 Pa m. Dividing this quantity by the reference stress provides the characteristic length $L_{\mathrm{R}}$ of $10^{-8} \mathrm{~m}$.

The only physical time scale discussed so far enters the kinetic law. Here, we select the simplest kinetic law of the class (8) corresponding to a linear relation between the mass flux and the thermodynamic force with a phenomenological parameter $\mathscr{L}$. From the data of Rimstidt and Barnes (1980), the value $1.1 \times 10^{-14} \mathrm{~kg} \mathrm{~s} / \mathrm{m}^{4}$ is assigned to this parameter, at a temperature of $217^{\circ} \mathrm{C}$. This value is the characteristic $\mathscr{L}_{\mathrm{R}}$ which is used to define the characteristic time in Table 1 (equal approximately to a week). The dimensional analysis results in the following relation between dimensionless non-material velocity and thermodynamic force

$$
S_{N}=-\chi .
$$

This result is obtained with the assumption of small transformation which stipulates that the reference and current configurations are sufficiently close to disregard to first order the difference between $\mathrm{d} S$ and $\mathrm{d} S_{0}$ in Eq. (7). The dimensionless driving force introduced in Eq. (8) with the small transformation assumption reads

$$
\chi=\frac{1}{2}\left(K-\frac{2}{3} G\right)(\operatorname{tr} \varepsilon)^{2}+G \operatorname{tr}\left(\varepsilon^{2}\right)+(1+\operatorname{tr} \varepsilon)\left(p_{\mathrm{f}}-2 H\right)-\mu_{\mathrm{s}},
$$

in which $\varepsilon$ is the infinitesimal strain tensor. Note that Eq. (12) was obtained by replacing the ratio $\left(\tilde{\gamma} / \gamma_{\mathrm{R}}\right)$ by its value of one. Note also that the Helmholtz free energy of the solid phase under isothermal conditions is simply the elastic stored energy which is of same order as the variation in the mass density found in the numerator of the 
second term on the right-hand side of Eq. (8). This comparison justifies our choice to keep the inverse of the change in volume in Eq. (12) with the small transformation approximation. The importance of retaining this term in a linear stability analysis was discussed by Leroy and Heidug (1994) and was already clear from the work of Lehner and Bataille (1984/85).

\subsection{Weak formulation}

The last topic of this section concerns the presentation of the weak formulation of the initial- and boundary-value problem. The elastic material occupies the volume $\Omega(t)$ with boundary $\partial \Omega(t)$ and it is in contact with a fluid phase over the interface $\partial \Omega_{\mathrm{I}}(t)$. The phase transformation acts on this interface $\partial \Omega_{\mathrm{I}}(t)$ which evolves with time and its position constitutes one of the unknowns of the problem. Note that the fluid phase is in chemical and mechanical equilibrium and thus always homogeneous for both open and closed systems. For this reason, a numerical discretization is only required for the solid phase.

At any time $t$ at which the shape of the solid-phase reference configuration is known, the principle of virtual work provides the mechanical equilibrium condition

$$
\int_{\Omega(t)}\left(K-\frac{2}{3} G\right) \operatorname{tr}(\boldsymbol{\varepsilon}) \nabla \cdot \delta \mathbf{u}+2 G \boldsymbol{\varepsilon}: \nabla \delta \mathbf{u} \mathrm{d} V+\int_{\partial \Omega_{\mathrm{I}}(t)} \delta \mathbf{u} \cdot \mathbf{N}\left(2 H-p_{\mathrm{f}}\right) \mathrm{d} S=0,
$$

in which $\delta \mathbf{u}$ and $\nabla$ are a kinematically admissible virtual displacement and the gradient operator, respectively. Note that the applied traction vector over the solid-fluid interface accounts for the effects of surface tension according to Laplace's conditions (4).

The solution of Eq. (13) provides, for a known position of the solid-fluid interface, the material displacement $\mathbf{u}$ over the physical domain. This information is then used to determine the Helmholtz free energy of the solid at the interface and thus to estimate the thermodynamic driving force (12) responsible for its migration. The non-material velocity of the interface is then provided by the kinetic law (11), which cannot be enforced pointwise if a numerical approximation is introduced. The error or residual is $\left(S_{N}^{h}+\chi\right)$ in which $S_{N}^{h}$ is the numerical approximation of the speed of propagation. We impose here that the weighted average of this residual integrated over the interface vanishes

$$
\int_{\partial \Omega_{\mathrm{I}}(t)}\left(S_{N}^{h}+\frac{1}{2}\left(K-\frac{2}{3} G\right)(\operatorname{tr} \boldsymbol{\varepsilon})^{2}+G \operatorname{tr}\left(\varepsilon^{2}\right)+(1+\operatorname{tr} \boldsymbol{\varepsilon})\left(p_{\mathrm{f}}-2 H\right)-\mu_{\mathrm{S}}\right) \varphi \mathrm{d} S=0
$$

for some appropriate choice of the weight function $\varphi$. The solution of Eqs. (13) and (14) provides the two unknowns of the problem which are the displacement over the whole domain and the non-material velocity $S_{N}^{h}$ of the solid-fluid interface. The numerical scheme designed for solving the evolution problem and based on this weak formulation is found in the next section. 


\section{A class of finite elements for IPS}

The need for a special finite-element scheme to capture the evolution with time of the solid-fluid interface can be motivated from the interpretation of numerous linear stability analyses found in the literature (Srolovitz, 1989; Leroy and Heidug, 1994). The results presented in Appendix $\mathrm{C}$ on the stability of a rectangular block point to the same conclusion. An interface between a stressed solid and a fluid in mechanical equilibrium is destabilized if the stress component in the direction tangent to the interface differs from the fluid pressure. The stabilizing factor, due to surface tension, is proportional to the mean curvature. The way this curvature is estimated over the discretized interface is now discussed. A classical finite-element scheme relies on a $C_{0}$ interpolation rendering the tangent vector along the solid-fluid interface discontinuous across elements. Any stress concentration close to the element boundaries is then destabilizing and cannot be balanced by surface tension if the measure of curvature does not account for the discontinuity at element boundaries. This problem was found to be alleviated by a $C_{1}$ interpolation of the solid-fluid interface. A rapid local variation of the tangent affects the curvature of the elements in the neighborhood and thus triggers the stabilizing influence of surface tension. A similar idea was used by Yang and Srolovitz (1994) in a boundary integral scheme.

This section is made of three parts. The first describes the class of elements considered with a $C_{1}$ interpolation along the solid-fluid interface. The second deals with the time discretization and the solution algorithm. The basic unknowns are the material displacements (and its surface gradient) and the position (including the tangent) of the interface. The third part concerns the mesh adaptation which is used to parameterize the interface as well as to modify the mesh over the reference configuration whose boundary evolves with time. Proof of convergence of the numerical scheme is discussed in Appendix B. The reader most interested by the physics of the problem can skip this section altogether. Note that although the governing equations were defined in the previous section in 3 dimensions, the finite-element scheme to be presented is restricted to the plane. Extension to $3 \mathrm{D}$ while straightforward from the theory remains a major technical challenge.

\subsection{Elements with $C_{1}$ interpolation on part of their boundary}

Our objective is the construction of a finite element having $C_{1}$ continuity on one of its sides, if located at the solid-fluid interface, and having the usual $C_{0}$ properties in the bulk of the solid phase. The starting point is the interpolation along the interface which is divided in a number of elements. Any point $\mathbf{X}$ on that interface is located on the boundary of an element (Fig. 1) and has its position interpolated by

$$
\mathbf{X}\left(\xi_{1}\right)=\sum_{a=1}^{4} H_{a}\left(\xi_{1}\right) \tilde{\mathbf{Y}}_{a}
$$

in which $\tilde{\mathbf{Y}}_{a}$ are generalized nodal position vectors comprising 2 nodal positions $\left(\mathbf{Y}_{1}, \mathbf{Y}_{2}\right)$ and two tangent vectors $\left(\lambda_{1} \mathbf{T}_{1}, \lambda_{2} \mathbf{T}_{2}\right)$, having for norm $\lambda_{1}$ and $\lambda_{2}$. These functions 

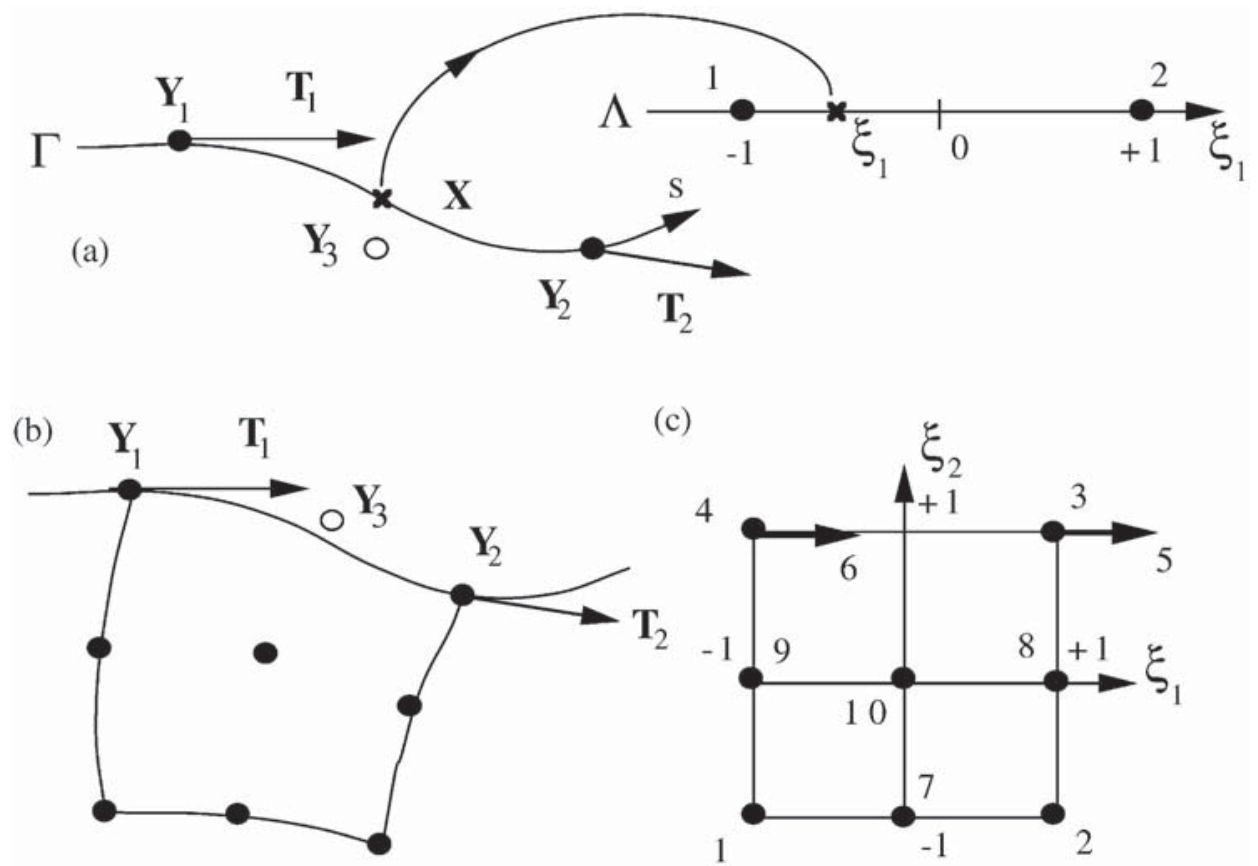

Fig. 1. The finite-element discretization. The $C_{1}$ discretization of the interface (a) is realized with Hermite polynomials. This interpolation is coupled to Lagrange polynomials to construct a finite-element with $C_{0}$ continuity in the bulk and $C_{1}$ on the interface (b). The local node numbering of the element is presented in the computational space in (c).

$H_{a}\left(\xi_{1}\right)$ are first-order Hermite polynomials of the natural coordinate $\xi_{1}$. The scalars $\lambda_{1}$ and $\lambda_{2}$ are the Jacobian at $\xi_{1}$ equal to -1 and +1 of the transformation between the curve $\Gamma(s)$ of arc length $s$ and the $\Lambda\left(\xi_{1}\right)$ axis (Fig. 1a). These scalars are part of the interface parameterization and it is proposed to determine them from constraints of the type

$$
\lambda_{\alpha}=\mathscr{G}_{\alpha}\left(\tilde{\mathbf{Y}}_{a}, \tilde{\mathbf{Y}}_{3}\right)
$$

The two unknowns $\lambda_{\alpha}$ in Eq. (16) are defined in terms of an extra node $\mathbf{Y}_{3}$, shown as an open circle in Fig. 1a. For example, the function $\mathscr{G}_{\alpha}$ could be constructed such that $\mathbf{Y}_{3}$ be positioned on the interface at the coordinate $\xi_{1}$ equal zero. A discussion of this particular choice is found in Ghoussoub (2000).

Interpolation (15) should be compatible with the finite-element discretization over the solid phase having part of its boundary on that interface (Fig. 1b). For that purpose, a special class of elements is constructed having this specific property. The elements have their shape functions constructed by combining Lagrange's and Hermite's polynomials. Their exact expressions are found in Appendix B and it suffices here to know that the interpolation over the element is given by

$$
\mathbf{X}\left(\xi_{1}, \xi_{2}\right)=\sum_{a=1}^{N} N_{a}\left(\xi_{1}, \xi_{2}\right) \tilde{\mathbf{X}}_{a}
$$

in which $N_{a}$ and $\tilde{\mathbf{X}}_{a}$ are the shape functions and the generalized nodal coordinates (Fig. 1b). The list of generalized coordinates is $\left(\mathbf{X}_{1}, \mathbf{X}_{2}, \mathbf{Y}_{2}, \mathbf{Y}_{1}, \lambda_{2} \mathbf{T}_{2}, \lambda_{1} \mathbf{T}_{1}, \mathbf{X}_{7}, \mathbf{X}_{8}\right.$, 
$\left.\mathbf{X}_{9}, \mathbf{X}_{10}\right)$ if the number of nodes $N$ is set to 10 and according to the connectivity defined in Fig. 1c.

The next step in the construction of the finite-element scheme concerns the interpolation of the displacement field. Here, we favor the idea of an isoparametric element in which displacement and position interpolation are thus identical. The collection of generalized nodal displacement $\tilde{\mathbf{u}}_{a}$ includes the material displacement as well as its surface gradient: $\left(\mathbf{u}_{1}, \mathbf{u}_{2}, \mathbf{u}_{3}, \mathbf{u}_{4}, \lambda_{2}\left(\nabla_{\Gamma} \mathbf{u}\right)_{2}, \lambda_{1}\left(\nabla_{\Gamma} \mathbf{u}\right)_{1}\right)$ for the basic 4 noded element and with $\nabla_{\Gamma}$ defining the surface gradient over the interface $\Gamma$. In view of the definition of the scalars $\lambda_{\alpha}(\alpha=1,2)$ as Jacobians of the transformation between $\Gamma(s)$ and $\Lambda\left(\xi_{1}\right)$ at $\xi_{1}$ equal to -1 and +1 , the surface gradient $\left(\nabla_{\Gamma} \mathbf{u}\right)_{\alpha}$ times $\lambda_{\alpha}$ could be replaced by $\left(\nabla_{\Lambda} \mathbf{u}\right)_{\alpha}$, the gradient of the displacement over the $\Lambda$-axis.

\subsection{The mesh adaptation scheme}

The evolution in time of the solid-fluid interface requires the modification of the finite-element discretization of the domain occupied by the solid phase. The solution adopted to define the mesh over the reference configuration $\Omega_{0}(t)$ relies on a boundary conforming mapping. It is a variational method in which the boundaries of the reference configuration, the physical domain, coincide with the transformed coordinate lines of the computational domain $\Pi$ which are equi-potential. This method, based on the work of Brackbill and Saltzman (1982), aims to optimize three properties of the discretization which are its smoothness, the orthogonality of the coordinate lines and the variation or concentration of the elements in regions of interest. The $\xi_{\alpha}$-coordinate line mapped on the computational domain has a gradient defined by the vector $\partial \xi_{\alpha} / \partial X_{i}$. Smoothness of the coordinate lines spacing is then measured by the sum of the squares of the norm of the gradient vector for the two lines

$$
I_{\mathrm{S}}=\int_{\Omega_{0}} \frac{\partial \xi_{\alpha}}{\partial X_{j}} \frac{\partial \xi_{\alpha}}{\partial X_{j}} \mathrm{~d} V .
$$

Orthogonality is estimated by the square of the scalar product of the gradient between the $\xi_{1}$ - and $\xi_{2}$-lines

$$
I_{\mathrm{O}}=\int_{\Omega_{0}}\left(\frac{\partial \xi_{1}}{\partial X_{j}} \frac{\partial \xi_{2}}{\partial X_{j}}\right)^{2} J_{\Pi}^{3} \mathrm{~d} V,
$$

in which $J_{\Pi}$ is the Jacobian of the transformation from the computational to the physical domain. The concentration of the discretization is measured by

$$
I_{\mathrm{C}}=\int_{\Omega_{0}} \omega J_{\Pi} \mathrm{d} V,
$$

with the introduction of $\omega$, a function of the Helmholtz free energy (Ghoussoub, 2000).

The Euler-Lagrange equations of the adaptation scheme are then obtained by minimizing the linear combination of the 3 potentials in Eqs. (18)-(20)

$$
I_{\mathrm{S}}+\lambda_{\mathrm{O}} I_{\mathrm{O}}+\lambda_{\mathrm{C}} I_{\mathrm{C}},
$$


where $\lambda_{\mathrm{O}}$ and $\lambda_{\mathrm{C}}$ are two scalars. These two scalars could be seen as Lagrange multipliers since $I_{\mathrm{O}}$ and $I_{\mathrm{C}}$ do not lead to elliptic operators at a difference with $I_{\mathrm{S}}$ (Brackbill and Saltzman, 1982). Furthermore, the selection of these scalars is dictated by the fact that the 3 potentials in Eqs. (18)-(20) do not share the same dimension, as pointed out by Kreis et al. (1986). If $I_{\mathrm{S}}$ is of order 1 , then $I_{\mathrm{O}}$ and $I_{\mathrm{C}}$ are of order $h$ and $\omega h$, respectively, in terms of the parameter $h$ which is the characteristic element size. Appropriate scaling in Eq. (21) requires $\lambda_{\mathrm{O}}$ and $\lambda_{\mathrm{C}}$ to be of order $h^{-4}$ and $\omega^{-1} h^{-4}$, respectively.

Various difficulties arise with the use of this class of variational methods for adapting numerical discretization which are discussed in Christodoulou and Scriven (1992) and Tsiveriotis and Brown (1992). The latter contribution is closer to our problem since it was concerned with the migration of solidification fronts. Among those numerical problems, we should mention the difficulty to concentrate $\xi_{\alpha}$-coordinate lines parallel to convex boundaries compared to concave boundaries and the absence of parameterization of the curved solid-fluid interface. Solutions to these problems and further information on the adaptation scheme, including the linearized version of the Euler-Lagrange equations for the class of finite elements proposed above, are found in Ghoussoub (2000). The values chosen for every parameter of the mesh adaptation in the calculations presented in this paper are also found in that reference.

\subsection{Time discretization and solution strategy}

Time is discretized and it is assumed that at time $t_{n}$ the reference configuration $\Omega_{\mathrm{O}}\left(t_{n}\right)$ is known. The four steps now presented summarize the procedure proposed to compute the next position of the solid-fluid interface at time $t_{n+1}$.

First, knowing the reference configuration at time $t_{n}$ one determines the material displacement through the application of the principle of virtual work (13). The strain and stress are then known over the domain $\Omega_{\mathrm{O}}\left(t_{n}\right)$ and, in particular, over the solid interface $\partial \Omega_{\mathrm{I}}\left(t_{n}\right)$ where the thermodynamic force has to be computed. In a second step, the weak form of the kinetic law (14) is solved to provide the non-material velocity $S_{N}^{h}\left(t_{n}\right)$. The position of the solid-fluid interface is updated in a third step by an explicit scheme based on the non-material velocities just computed. It remains in the fourth step to modify the parameterization of the interface and, subsequently, to adapt the finite-element discretization over the solid phase. These two mesh adaptations are not conducted at every time step and a simple criterion is proposed to activate this last part of the procedure: the mesh is adapted over the volume or the interface only if there is at least one element whose area increases by more than a certain percentage, set to 3 , for most of the results to be presented.

The accuracy of the explicit scheme has been tested by comparing several choices of time step for the same problem. It has been found that the solution is accurate if during a time step any element does not have a relative change in its surface exceeding $10^{-2 \%}$. It is clear that an implicit scheme could be more interesting since it would allow larger time step. It would require the simultaneous resolution of the mesh adaptation scheme, the weak form of the kinetic law and the principle of virtual work. However, the implicit scheme does not have a symmetric tangent operator in the absence of a 
variational formulation for the coupled problem. Furthermore, the increase in number of nodal unknowns compared to our staggered algorithm may cancel the benefit of an implicit scheme.

The final word on the numerical scheme concerns the case of a constrained fluid phase for a closed system. In that instance, the solution procedure has to be amended to permit as a fifth step the computation of the chemical potential at time $t_{n+1}$. For that purpose, the new volume of the inclusion is first computed as well as the total mass present in the solid phase. The difference in mass with respect to the previous time step determines the variation in solute mass in the fluid phase and thus the current concentration $C_{\mathrm{S}}$. The pore fluid pressure is then determined by solving the non-linear equation (10). Note that the time step is always smaller for the study of closed systems in order to avoid spurious fluctuations in pore pressure.

\section{Results}

The main objective of this paper is to gain a better understanding of the evolution of the grain boundary structure during a loading cycle. In Fig. 2, a transmission optical micrograph of a grain boundary in rock salt after deformation by IPS in the laboratory is presented (result obtained in the HPT Laboratory, Faculty of Earth Sciences, Utrecht University). The grain boundary shows the typical island and channel structure discussed before. The channels are tubular cavities, about $3 \mu \mathrm{m}$ in diameter, which were fluid filled during the experiments. Note also the existence of isolated inclusions within the grain boundary. The contact made by the islands are likely to

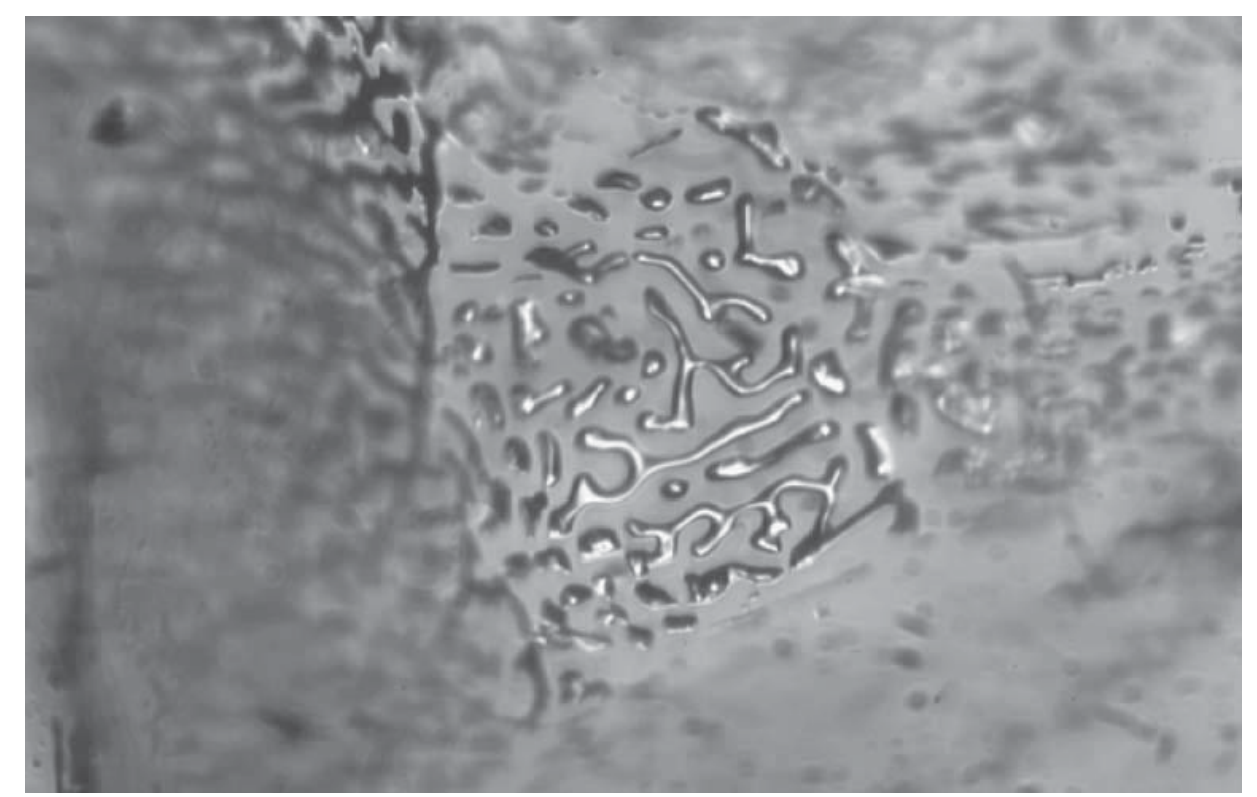

Fig. 2. Transmission optical micrograph showing fluid inclusions within grain boundaries in experimentally deformed rocksalt. The salt is synthetically produced and contains about $50 \mathrm{ppm}$ of water. It was deformed at a temperature of $200^{\circ} \mathrm{C}$, a confining pressure of $50 \mathrm{MPa}$ and a constant strain rate of $5 \times 10^{-7} \mathrm{~s}^{-1}$. The tubular inclusions are typically $3 \mu \mathrm{m}$ in diameter. Results were obtained in the HPT Laboratory, Faculty of Sciences, Utrecht University, The Netherlands and presented with the kind permission of Prof. C.J. Spiers. 


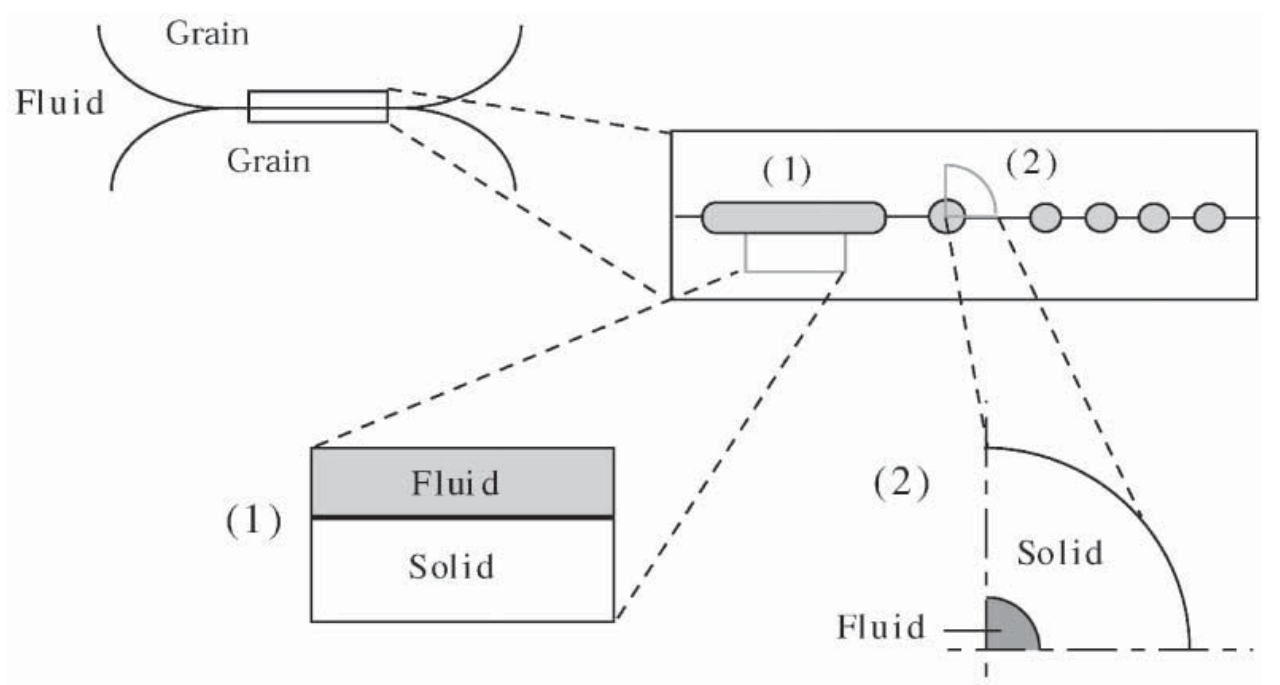

Fig. 3. Schematic diagram of a grain boundary and of two different features of its internal structure. They lead to the proposition of two initial- and boundary-value problems. The first problem (1) consists of a rectangular block sustaining a plane-strain compression and exposed on its top side to the fluid phase. The second problem geometry (2) is a cylindrical cavity, a quarter of the structure being studied for reason of symmetry.

be dry regions even though the existence of thin films, especially during the experiments, cannot be ruled out. This experimental observation motivates the proposition of the idealized grain boundary depicted in Fig. 3. Two features in this idealized grain boundary are sketched. They will be at the basis of two initial- and boundary-value problems proposed to model the time evolution of an idealized grain boundary.

The first structure considered presupposes that a thin fluid film penetrates at least partly the grain boundary and provides an efficient channel for the transfer of any dissolved material to the pore fluid. The corresponding model problem is referred to as problem 1 in Fig. 3 and consists of a rectangular block in contact on its top side with the fluid phase. Only the case of a constant solute chemical potential is explored, corresponding to the definition of an open system in Section 2.

The second microstructural feature found in Fig. 3 is a cylindrical fluid-filled tube trapped within the grain boundary. This geometry is of course reminiscent of the channel structure observed in Fig. 2. The model problem extracted from that microstructure and labeled problem 2 in Fig. 3 consists of a cylindrical cavity in an elastic solid sustaining a remote compression normal to the grain boundary. The energy associated with the solid-solid contact is disregarded and a circular cross section is the equilibrium shape. For reasons of symmetry, only a quarter of the structure is studied. To discuss the influence of the fluid phase, both the open and closed systems are considered. For open systems, the solute chemical potential is kept constant and takes the value necessary to have thermodynamic equilibrium under the initially hydrostatic stress conditions. For closed systems, the fluid phase is assumed isolated within the grain boundary and the chemical potential varies according to the fluid pressure and the solute concentration calculated in the plane of study. 


\subsection{The rectangular block}

The first problem considered consists of the rectangular block, thickness $H$ and length $L$, shown in Fig. 4. The solid-fluid interface $\mathrm{BB}^{\prime}$ is initially plane and the compression of the block is due to a force $F$ or a displacement $U$ prescribed on the right lateral boundary $\mathrm{A}^{\prime} \mathrm{B}^{\prime}$. The force is imposed by a rigid plate of constant length denoted $H_{G}$. The thickness $H$ is always less than $H_{G}$ and the fluid pressure acts on the top of the plate over the length $H_{G}-H$. This particular choice was found convenient for the linear stability analysis. Conditions of plane strain deformation prevail in the elastic block. The numerical data are summarized in Table 2. The fundamental solution is first discussed prior to the analysis of the initial morphology evolution of the solid-fluid interface when its position is perturbed by a cosine wave of small amplitude. The long term evolution of this type of perturbation is then considered.

The fundamental solution of this problem corresponds to an initially rectangular block whose shape remains homothetic at all times. The rate of change of the thickness $H(t)$ is equal to the non-material velocity $S_{N}$ of the solid-fluid interface, which, according to the linear kinetic law (11), reads

$$
\dot{H}(t)=-\frac{1-v}{4 G}\left(\sigma_{0}+p_{\mathrm{f}}\right)^{2}-p_{\mathrm{f}}+\mu_{\mathrm{S}},
$$

in which $v$ and $\sigma_{0}$ are Poisson's ratio and the uniform stress acting in the $x$-direction, respectively. This stress $\sigma_{0}$ depends on the type of boundary conditions

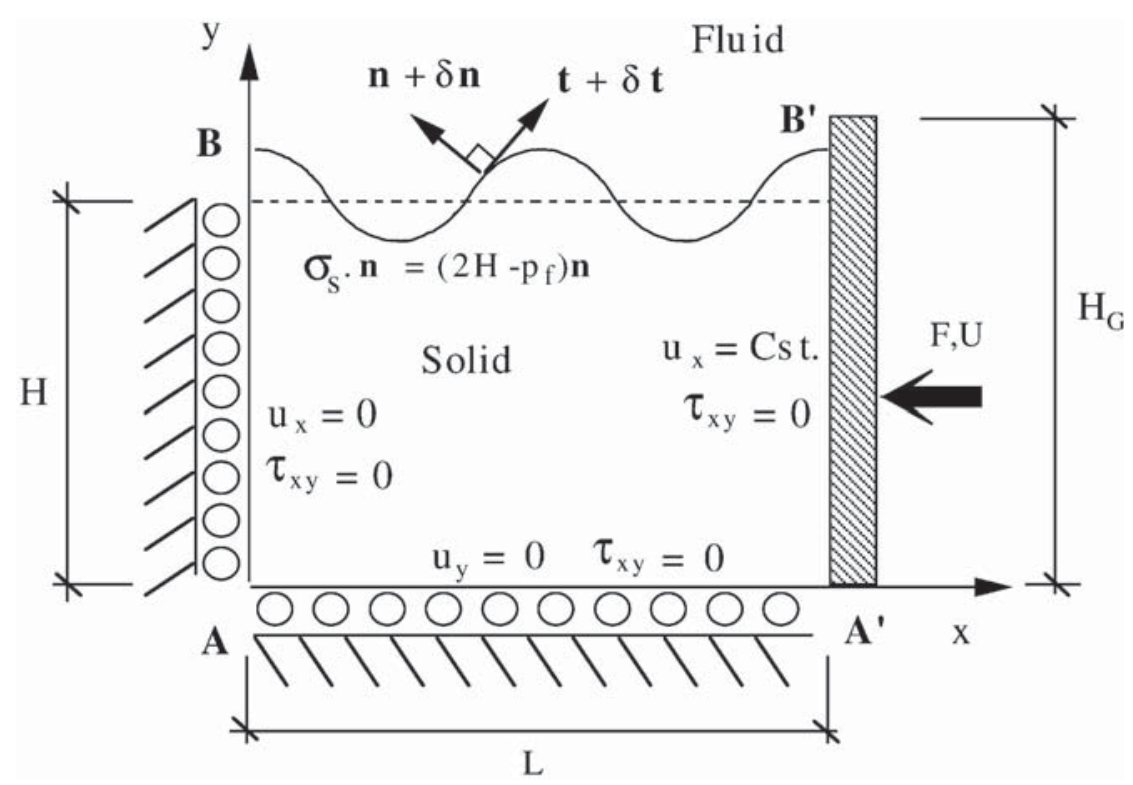

Fig. 4. The first model problem consists of a rectangular block in contact on its top side with the fluid phase. The wall $\mathrm{A}^{\prime} \mathrm{B}^{\prime}$ is displaced uniformly to produce a compression by either keeping the force $F$ constant or prescribing the displacement $U$. The boundary conditions on the two sides $\mathrm{AB}$ and $\mathrm{AA}^{\prime}$ consist of no normal displacement and, in the absence of friction, of a zero shear stress. Laplace conditions (Eq. (4) in the text) are imposed on the solid-fluid interface. 
Table 2

Physical constants, geometrical parameters and loading conditions used for the analysis of the rectangular block, model problem 1

\begin{tabular}{llll}
\hline Parameter & Unit & $\begin{array}{l}\text { Reference } \\
\text { quantity }\end{array}$ & $\begin{array}{l}\text { Dimensionless } \\
\text { value }\end{array}$ \\
\hline Young's modulus $E$ & $\mathrm{~Pa}$ & $\sigma_{\mathrm{R}}$ & 8200 \\
Elasticity modulus in shear $G$ & $\mathrm{~Pa}$ & $\sigma_{\mathrm{R}}$ & 3000 \\
Surface tension $\gamma$ & $\mathrm{Pa} \mathrm{m}$ & $\gamma_{\mathrm{R}}$ & 1 \\
Constant solute chemical potential $\mu_{\mathrm{S}}$ & $\mathrm{Pa} \mathrm{m} / \mathrm{kg}$ & $\sigma_{\mathrm{R}} / \rho_{\mathrm{R}}$ & {$[3.012 ; 3.04]$} \\
Solid phase density $\rho_{0}$ & $\mathrm{~kg} / \mathrm{m}^{3}$ & $\rho_{\mathrm{R}}$ & 1 \\
Kinetics constant $\mathscr{L}$ & $\mathrm{kg} \mathrm{s} / \mathrm{m}^{4}$ & $\mathscr{L}_{\mathrm{R}}$ & 1 \\
Initial thickness $H=L$ & $\mathrm{~m}$ & $L_{\mathrm{R}}$ & 100,500 or 1000 \\
Constant or initial fluid pressure, $p_{\mathrm{f}}$ or $p_{\mathrm{f}}^{0}$ & $\mathrm{~Pa}$ & $\sigma_{\mathrm{R}}$ & 3 \\
Initial stress $\sigma_{0}$ & $\mathrm{~Pa}$ & $\sigma_{\mathrm{R}}$ & {$[-22 ;-18]$} \\
\hline
\end{tabular}

according to

$$
\begin{aligned}
\sigma_{0} & =\frac{2 G U}{1-v}-p_{\mathrm{f}} \frac{v}{1-v} \quad \text { for displ. control, } \\
\sigma_{0} & =-p_{\mathrm{f}}+\frac{F+p_{\mathrm{f}} H_{G}}{H} \quad \text { for force control. }
\end{aligned}
$$

The stress $\sigma_{0}$ is thus independent of $H$ for displacement control: the block thickness either increases or decreases at a constant rate, as illustrated by the two dotted curves in the phase diagram of Fig. 5, for the particular choice of $\sigma_{0}$ equal to $-p_{\mathrm{f}}$. A single value of $\sigma_{0}$ permitting thermodynamic equilibrium is found from Eqs. (22) and (23), if the pore fluid pressure is smaller than the dimensionless solute chemical potential. If that last condition is not met, $\dot{H}$ is always negative and dissolution occurs at a constant rate. If $p_{\mathrm{f}}$ is smaller than $\mu_{\mathrm{S}}$, dissolution or precipitation occurs depending on the effective stress $\left(\sigma_{0}+p_{\mathrm{f}}\right)$ found in Eq. (22). The importance of this effective stress $\left(\sigma_{0}+p_{\mathrm{f}}\right)$ will be confirmed by the stability analysis. For force control, the existence of an equilibrium state, marked by a solid dot in Fig. 5, also requires the pore pressure to be less than the dimensionless chemical potential of the solute $\mu_{\mathrm{S}}$. The importance of the order relation between $p_{\mathrm{f}}$ and $\mu_{\mathrm{S}}$ is illustrated by two solid curves for force control in Fig. 5. If the pore pressure is smaller than $\mu_{\mathrm{S}}$, the thermodynamic driving force does change sign and an equilibrium is reached for a thickness which is used as a reference value $H_{\text {Ref }}$. Fig. 5 is also instrumental in explaining the linear stability of an equilibrium following a homothetic perturbation of the block which remains thus rectangular in shape. For displacement control, this equilibrium is neutrally stable since the fundamental solution is independent of $H$. For force control, the equilibrium is unstable since any infinitesimal increase in the block thickness leads to a positive value of $\dot{H}$ which results in further increase in the block thickness. On the contrary, a perturbation which would reduce the block thickness renders the rate $\dot{H}$ negative and results in further reduction of the grain thickness, away from the equilibrium value. 


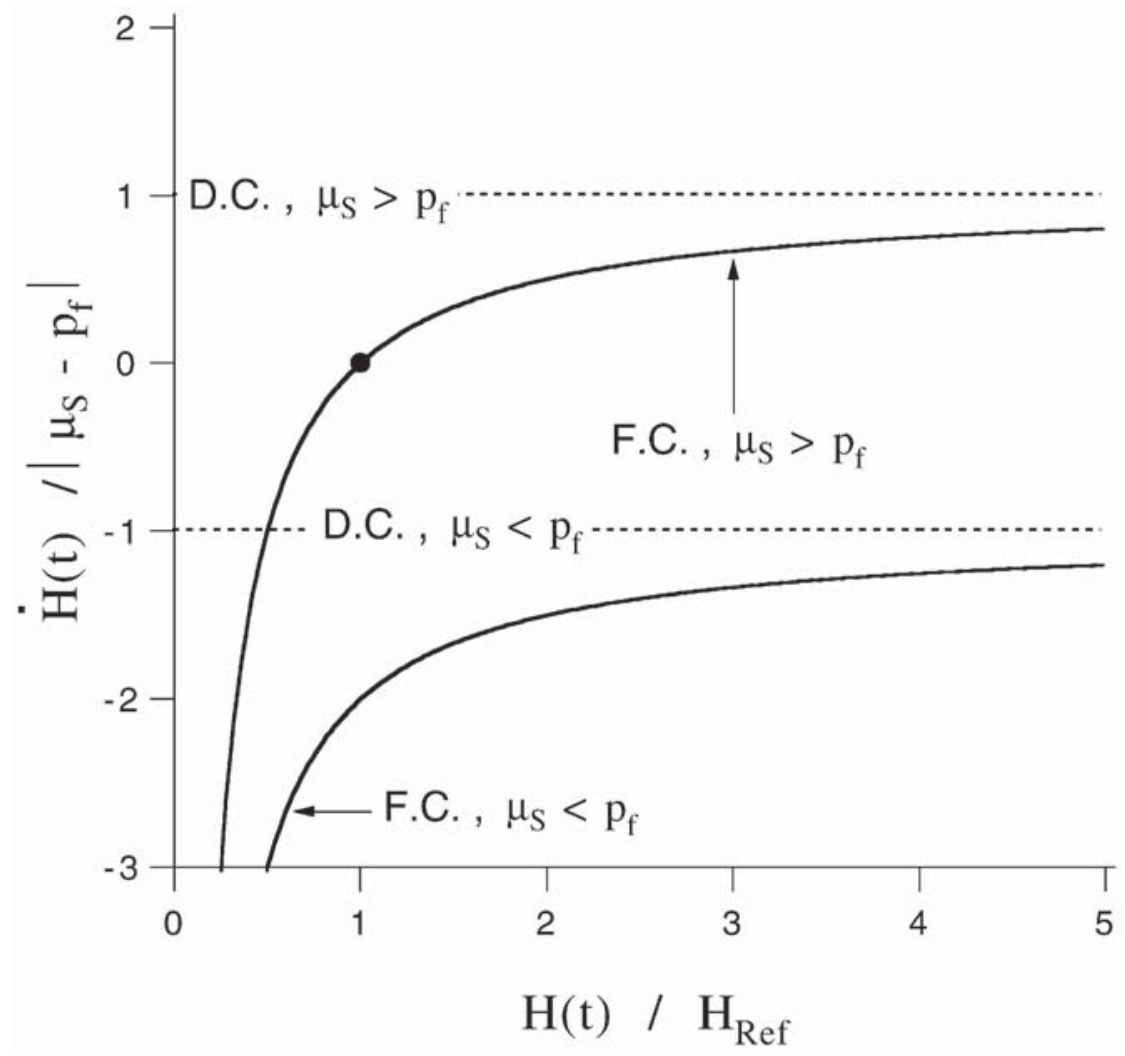

Fig. 5. Phase diagram for the fundamental solution of problem 1 defined by the rectangular block of thickness $H$ sketched in the previous figure. The rate of dissolution or precipitation is independent of $H$ in a displacement control test (DC), as shown by the dotted lines for the special case of $\sigma_{0}=-p_{\mathrm{f}}$. For the force control case (solid curves), an equilibrium (solid dot) to exist requires the dimensionless chemical potential to be larger than the pore pressure. Note that this equilibrium is unstable for a homothetic perturbation.

\subsubsection{Initial evolution and validation of the numerical scheme}

One of the reasons for studying problem 1 is that the initial evolution of wavy perturbations, of small amplitude compared to the block size, can be compared to the prediction of a linear stability analysis. This linear stability analysis, summarized in Appendix C, provides the following expression for the stability exponent:

$$
\lambda_{\xi}=-\xi^{2}+\xi\left(\sigma_{0}+p_{\mathrm{f}}\right)^{2} \frac{(1-v)}{G} \frac{\cosh ^{2}(\xi H)}{\cosh (\xi H) \sinh (\xi H)+\xi H},
$$

in which $\xi$ is the wavenumber of the perturbation, always a multiple of $\pi / L$. Note that the same result (24) applies for force and displacement control. If this stability exponent is positive for a given $\xi$ the solid-fluid interface is unstable. Conversely, a negative $\lambda_{\xi}$ for all $\xi$ defines a stable interface.

The analysis of this stability exponent is started with the first terms $-\xi^{2}$ on the right-hand side of Eq. (24) which is due to surface tension. It is negative and thus has a stabilizing effect for sufficiently large $\xi$ as can be seen from the four curves drawn in Fig. 6, for two choices of $H$ and of the compressive stress $\sigma_{0}$. The second term on the right-hand side of Eq. (24) is always positive or equal to zero and is thus destabilizing. It involves the product of $\xi$ and three factors. The first factor is 


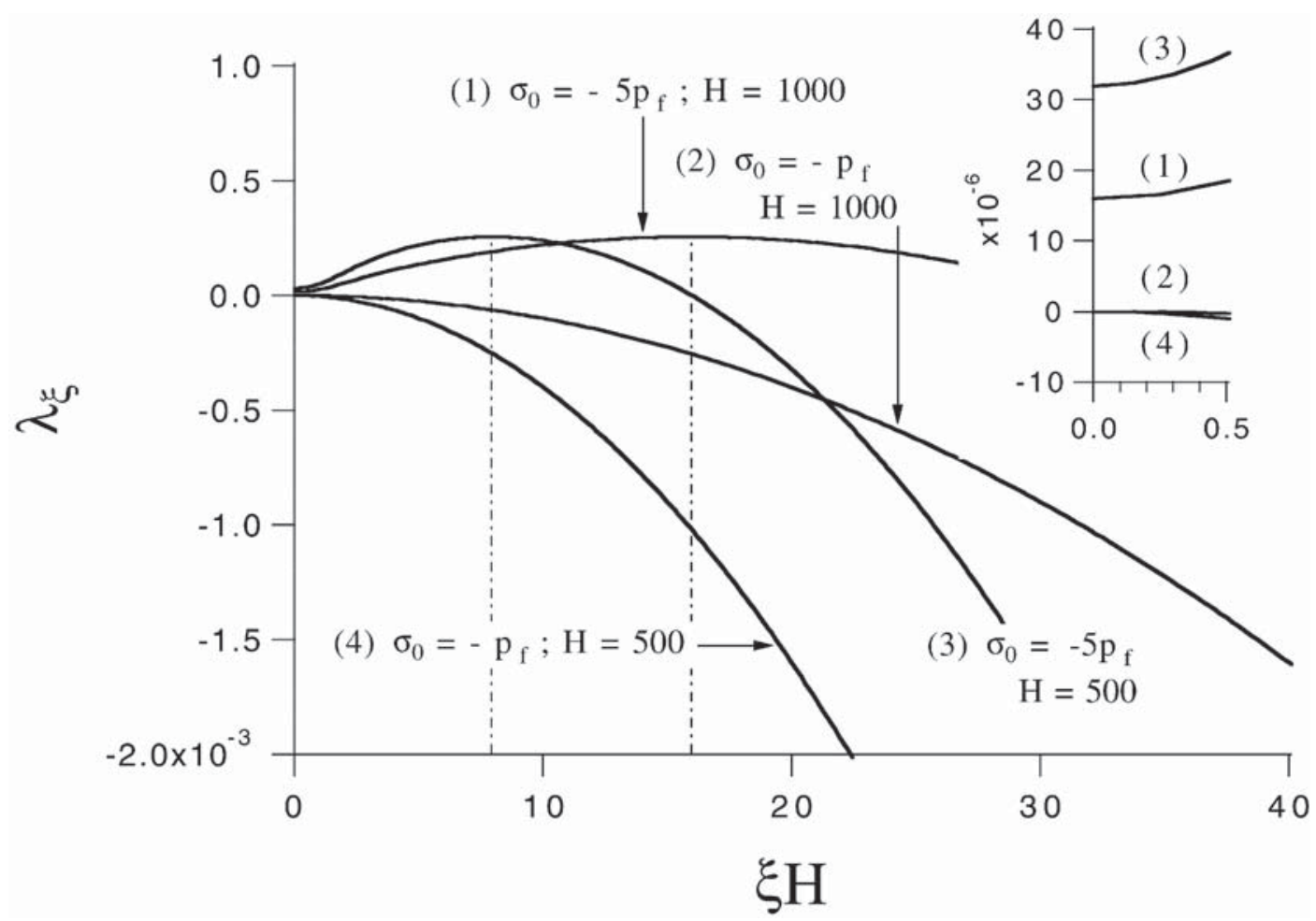

Fig. 6. Stability exponent as a function of the normalized wavenumber for two values of the thickness $H$ and of the compressive stress $\sigma_{0}$. The stability exponent is always negative, and the interface stable, if $\sigma_{0}$ is equal to minus the fluid pressure. If this condition is not met, there is a range of wavenumbers for which the stability exponent is positive and the interface is thus unstable. In the inset, the stability exponent is drawn in the long-wavelength limit for comparison with the analytical result obtained for $\xi=0$ (homothetic mode).

proportional to the square of the effective stress $\left(\sigma_{0}+p_{\mathrm{f}}\right)$ which needs to differ from zero for the interface to be unstable. As in Leroy and Heidug (1994), it is the difference between the interface-parallel compressive stress and the pore pressure which controls instability. If $\sigma_{0}$ is set to $-p_{\mathrm{f}}$, the stability exponent $\lambda_{\xi}$ is always negative, as seen in Fig. 6 (curve 4). There is thus for any $\sigma_{0}$ different from $-p_{\mathrm{f}}$ a range of $\xi$ for which the stability exponent is positive because surface tension effects are then of second order in $\xi$ and not sufficient to stabilize the interface. The wavenumber for which the stability exponent is maximum is called the dominant wavenumber. The present discussion has been conducted so far disregarding the influence of the third factor in the second term on the right-hand side of Eq. (24), which is due to the finite size of the studied domain and is a function of the normalized wavenumber $\xi H$. This geometrical function is close to one for values of $\xi H$ sufficiently large and its influence can then be disregarded. This can be seen from Fig. 6 by comparing the dominant normalized wavenumber for the same value of $\sigma_{0}=-5 p_{\mathrm{f}}$ but different block sizes (curves 1 and 3). According to Eq. (24), if the geometrical function of $\xi H$ is set to 1 , the dominant $\xi$ is then proportional to the square of the effective stress. The dominant dimensionless wavenumber in Fig. 6 should then scale by $H$. This is the case here with an error margin of $5 \%$, justifying the statement above on the influence of the finite geometry. This geometrical function of $\xi H$ has, however, a second salient feature: it is singular 


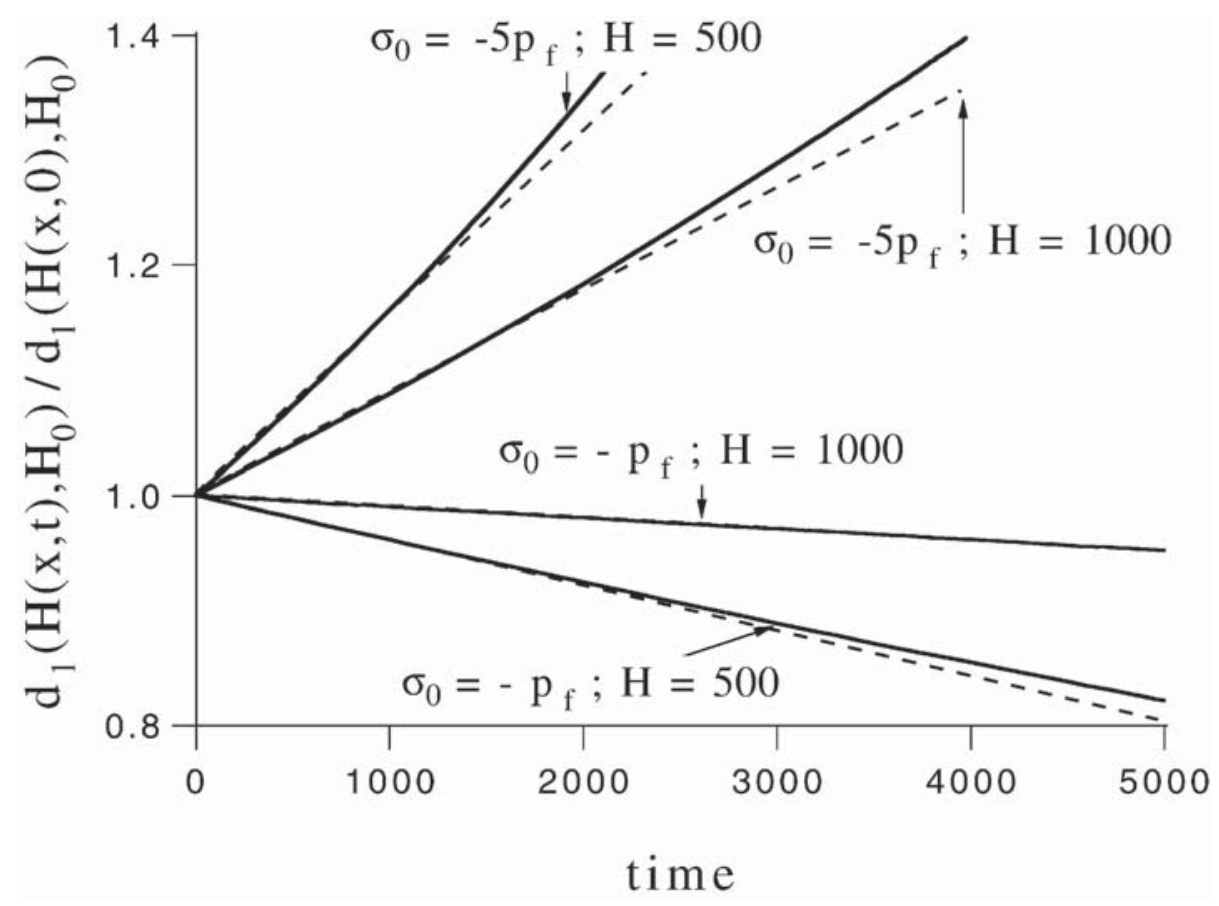

Fig. 7. The evolution in time of the perturbation size is analyzed by plotting the $d_{1}$ distance function (surface between the current solid-fluid interface and the initial flat perfect interface). This measure is normalized by the value of $d_{1}$ at time zero. The perturbation is a long-wavelength mode with $\xi=\pi / L$ and $L=H$. The agreement between the numerical solutions (solid curves) and the analytical predictions based on the linear stability analysis (dotted lines) validates the numerical scheme proposed.

for $\xi$ tending to zero leading to a finite value of $\lambda_{\xi}$ in that limit, as illustrated in the inset of Fig. 6. This limit is always positive if $\sigma_{0}$ differs from $-p_{\mathrm{f}}$ and inversely proportional to $H$. It is smaller than the maximum value of $\lambda_{\xi}$ confirming that the wavy perturbations are dominant, at least for the choice of $\sigma_{0}$ made in Fig. 6. A word of caution concerning this limit is now in order since the long-wavelength limit for $\lambda_{\xi}$ is found to be independent of the type of boundary conditions specified. This is at variance with the findings on the stability of the fundamental solution for a homothetic mode $(\xi=0)$ discussed earlier. The reason for that discrepancy, discussed in Appendix $\mathrm{C}$, is a singular limit in the boundary conditions of the stability analysis.

We now turn our attention to the comparison between the numerical solution and the linear stability prediction of the evolution of the solid-fluid interface perturbed by a cosine with wavenumber $\pi / L$ and amplitude $5 \%$ of $H$. The block has a square shape with $H$ set to either 500 or 1000 . The displacement is prescribed on the side resulting in a compressive stress $\sigma_{0}$, defined in Eq. (23), of either -1 or -5 times $p_{\mathrm{f}}$ for a perfect block. Results are presented in Fig. 7 in which the normalized distance function $d_{1}$ (surface between the actual position of the interface and the perfect interface at equilibrium) is plotted as a function of time (solid curves). For $\sigma_{0}=-p_{\mathrm{f}}$, the distance function is decreasing with time due to an evolution of the interface towards the flat equilibrium shape. To the contrary, for $\sigma_{0}=-5 p_{\mathrm{f}}$, the distance increases signaling the development of the instability. Note that in all cases, the rate of growth or decay of the perturbation coincides with the prediction of the linear stability analysis (dotted 


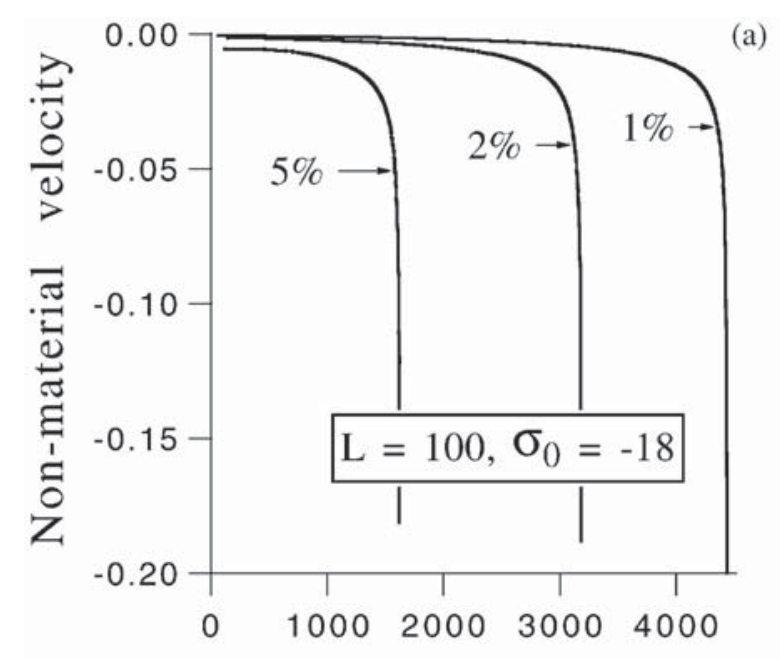

Time

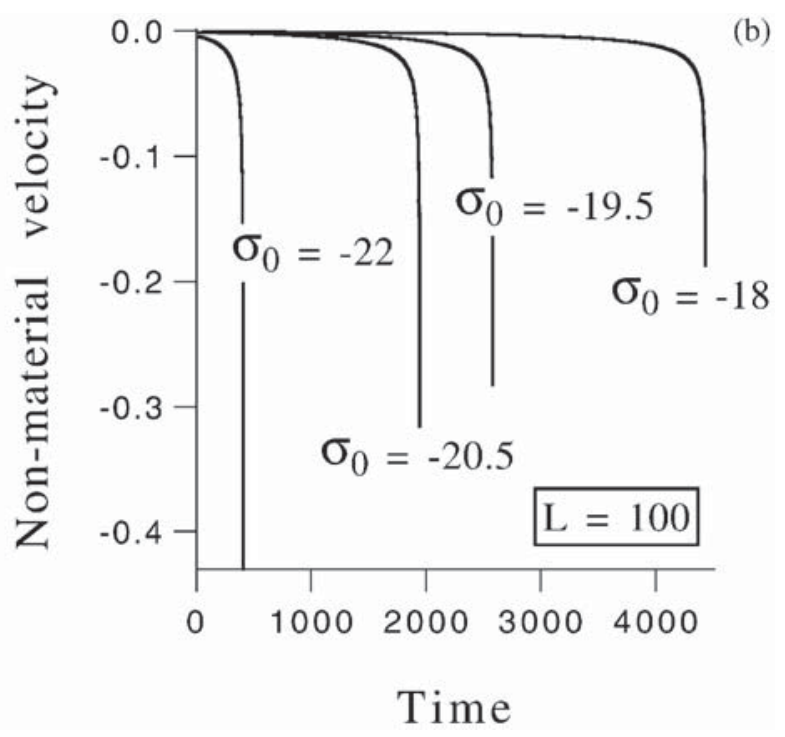

Fig. 8. The non-material velocity at the point along the interface having largest curvature as a function of time for the square block under compression presented in Fig. 4 and having a length of 100. In (a), the horizontal stress $\sigma_{0}$ is -18 and the long-wavelength imperfection has an amplitude of $1 \%, 2 \%$ and $5 \%$ times the block length. In (b), this amplitude is set to $1 \%$ and the stress is set between -18 and -22 . The reference stress is $10 \mathrm{MPa}$. A time of 1000 and a velocity of 1 represent 20.3 years and $0.5 \mu \mathrm{m} / \mathrm{yr}$, respectively.

lines) which are also found in Fig. 7. This agreement between numerical and analytical results validates the finite-element scheme proposed in the previous section.

\subsubsection{The long term evolution: formation of a dissolution pit}

The non-linear evolution of the solid-fluid interface of problem 1 is conducted first for a square block of length 100 and a displacement control resulting in a compressive stress $\sigma_{0}$ defined in Eq. (23) of -18 . The results of the computation are presented in Fig. $8 \mathrm{a}$ where the non-material velocity at the point of maximum curvature (right-hand side of the interface in Fig. 4) is a function of time for an amplitude of the imperfection set to $1 \%, 2 \%$ and $5 \%$ the block length. The main observation from this plot is the existence of two stages during the history of the velocity. The evolution is first rather slow during a period which lasts up to 4000 times the characteristic time. Then, the dissolution (negative velocity) becomes important and the non-material velocity reaches a value which is two orders of magnitude larger than in the first stage. The duration of stage 1 is affected by the imperfection size (Fig. 8a), as well as the value of the stress $\sigma_{0}$ (Fig. $8 \mathrm{~b}$ ). It is seen that an increase in the magnitude of $\sigma_{0}$ by $20 \%$ results in a reduction of the first stage duration by nearly an order of magnitude.

Similar results are presented as solid curves in Fig. 9 for a square block of length 500 , a stress $\sigma_{0}$ of -18 and an imperfection amplitude of $1 \%, 2 \%$ and $5 \%$ the block length. The transition between the two stages is sharper and, as before, the increase in the non-material velocity magnitude does not appear to become bounded. To understand this drastic evolution of the dissolution process, the position of the interface has been 


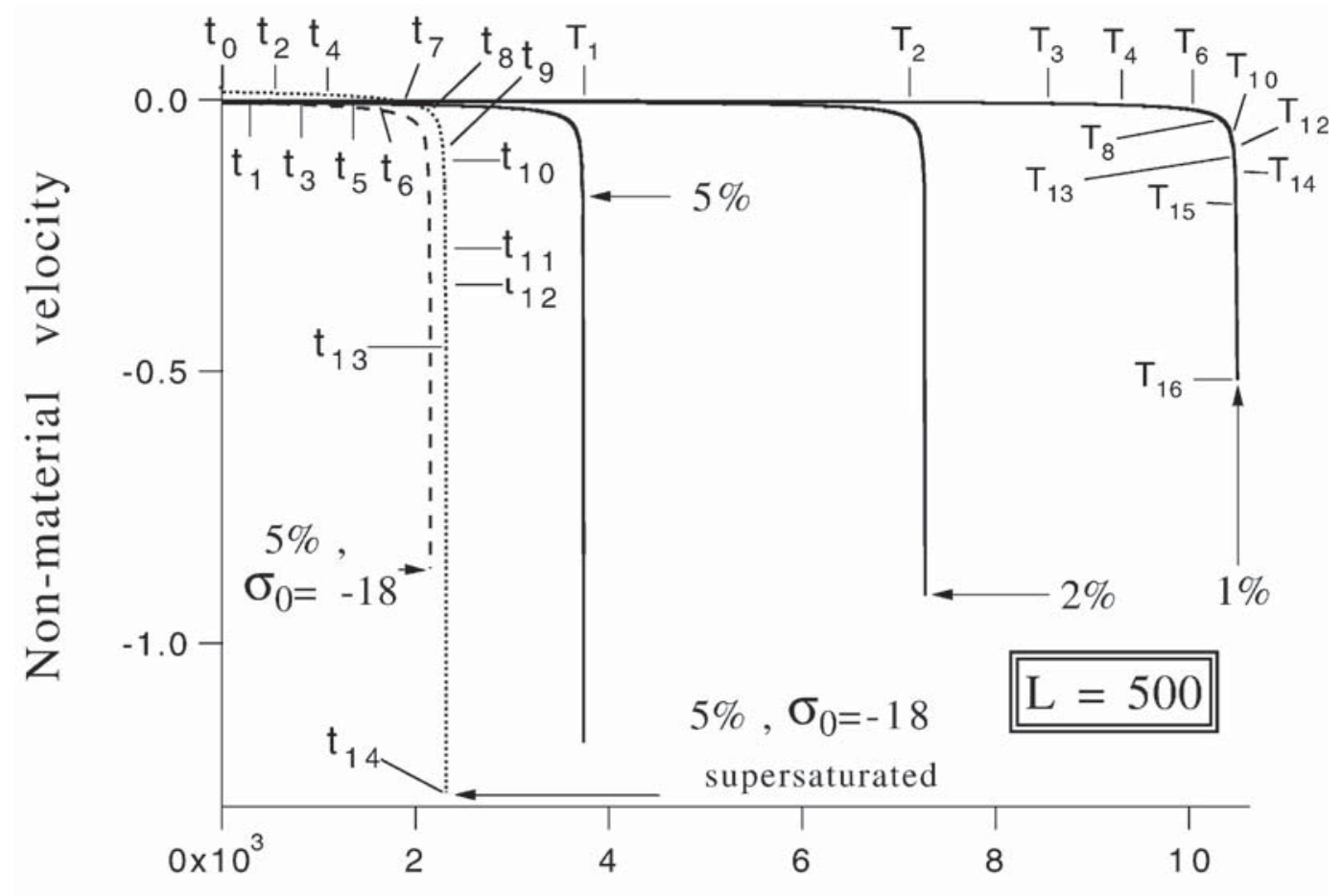

Time

Fig. 9. The evolution with time of the non-material velocity at the point along the interface having largest curvature, for the rectangular block of Fig. 4 having a length of 500, is presented for various imperfection sizes, a stress $\sigma_{0}$ of -15 (solid curves) and for a stress of -18 and an imperfection size set to $5 \%$ times the block length (dashed curve). The dotted curve is obtained for conditions similar to the ones selected for the dashed curve except for the chemical potential which is larger, resulting first in a deposition over the whole interface. A time of 1000 and a velocity of 1 represent 20.3 years and $0.5 \mu \mathrm{m} / \mathrm{yr}$, respectively.

plotted in Fig. 10 at 17 times identified by the letter $T$ in Fig. 9 for the particular case of an imperfection of $1 \%$. At time $T_{0}$, the interface has the cosine shape of the imperfection. Between time $T_{0}$ and time $T_{3}$, the morphology evolution of the interface is rather diffuse. The amplitude of the imperfection increases with deposition on the left and dissolution on the right. This is due to the variation in thermodynamic force along the interface resulting from a larger Helmholtz energy in the thinner section of the block. Note that between $T_{0}$ and $T_{3}$ there is a shift to the right of the inflection point along the interface which is where, initially, the thermodynamic equilibrium condition is met. This shift is due to an acceleration of the dissolution process compared to the deposition process. This acceleration is more obvious between time $T_{3}$ and $T_{6}$, just before the transition between stages 1 and 2 as defined above (Fig. 10). At time $T_{6}$, the morphology evolution is marked by a rapid dissolution over $10 \%$ of the block length. The rate of dissolution remains, however, of the same order as the rate of deposition. The important increase in dissolution occurs only after that time and takes place over a section which decreases in size, as seen in the inset of Fig. 10. Note that at time 16, the dissolution is now taking place over a length twice the characteristic length which is due to surface tension. However, the rate of dissolution keeps on increasing 


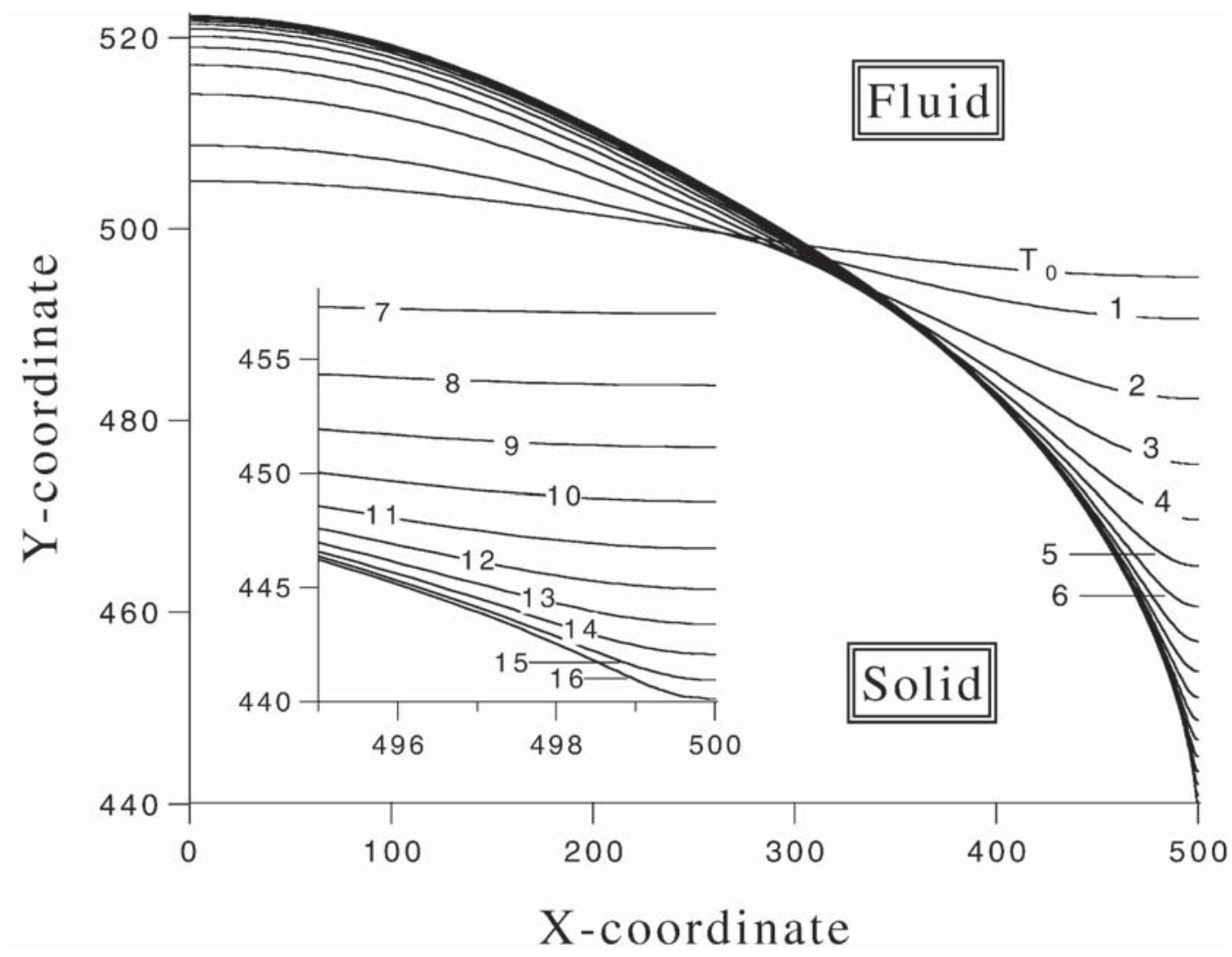

Fig. 10. The position of the interface at 17 times defined in Fig. 9 with the letter T. The zoom on the dissolution pit in the inset reveals that, at time 16, the dissolution is localized on a length scale which is of the same order as the characteristic length due to surface tension $\left(10^{-8} \mathrm{~m}\right)$.

and surface tension does not have the stabilizing influence found in the linear analysis. No physical mechanisms are accounted for here which could stop the formation of this sharp dissolution pit or cusp.

The reason for this unbounded growth can be explained by analyzing Fig. 11 which provides two types of information in the region around the tip of the dissolution pit. On the right-hand side of this figure, the finite-element mesh over the reference configuration at the end of the simulation is presented for the case of a block with length 100 , a stress $\sigma_{0}$ of -18 and a $2 \%$ imperfection. The $\mathrm{L}$ at the center provides the scale and the horizontal and vertical bars have for length 1.8 and $0.9\left(\times 10^{-8} \mathrm{~m}\right)$, respectively. These numbers allow to estimate the curvature at the tip of the cusp as well as the size of the smallest element. Note that this element has changed its size by a ratio of approximately 40, thanks to the mesh adaptation scheme. On the left-hand size of Fig. 11, 26 isocontours of equivalent Von Mises stress are presented in the range of $15-50(\times 10 \mathrm{MPa})$. In the zone close to the tip covered by nine elements, where isocontours are not plotted, this dimensionless equivalent stress reaches the value of $150(\times 10 \mathrm{MPa})$. The shape of those isocontours is reminiscent of the stress distribution at the tip of a sharp crack. They illustrate the importance of the stress concentration at the tip of the dissolution pit and the role played by the Helmholtz free energy. It is that part of the driving force which now controls the rate of dissolution. The simulations 


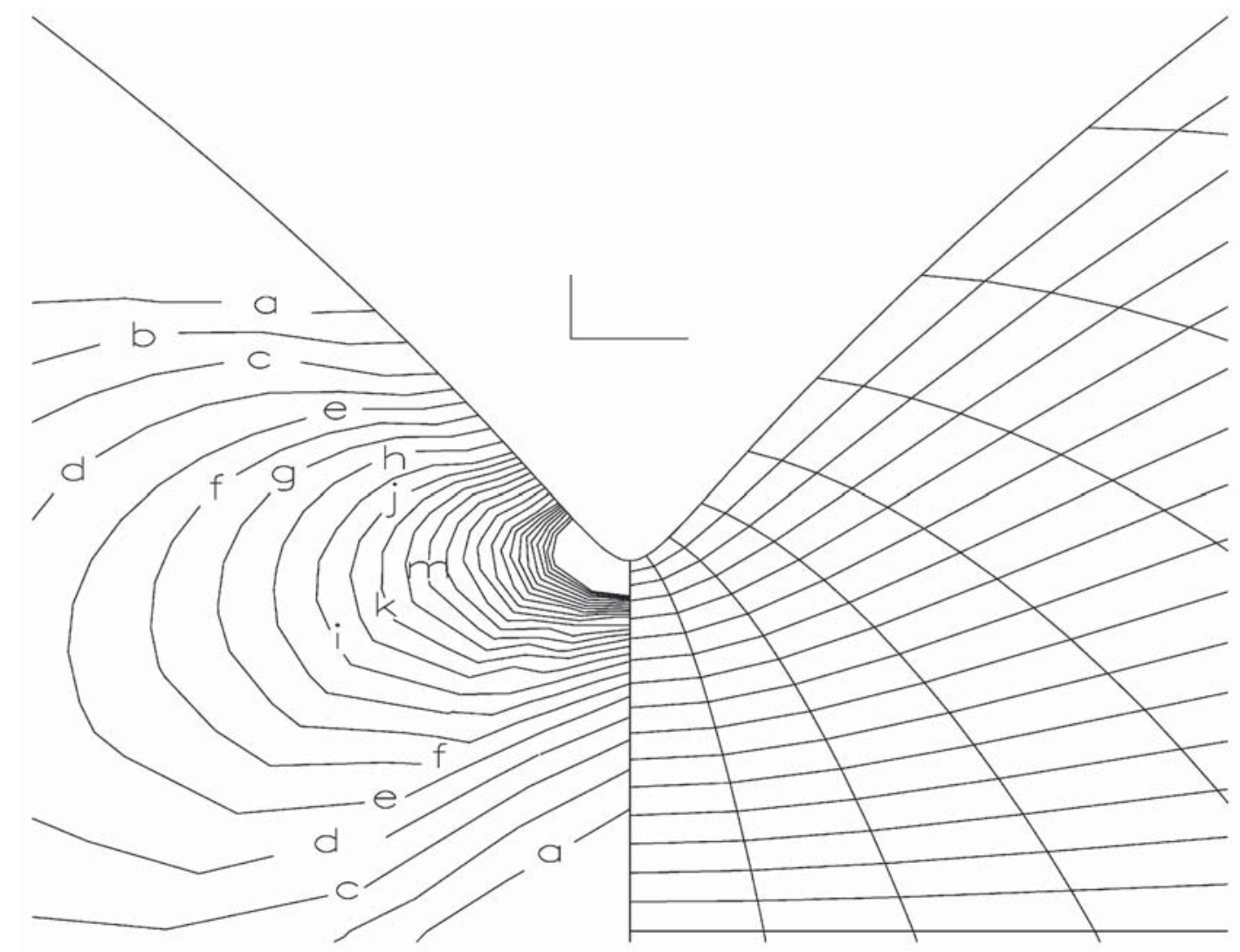

Fig. 11. The mesh and isocontours of equivalent Von Mises stress are presented in the tip region of the dissolution pit. The 26 isocontours $(\mathrm{a}-\mathrm{z})$ range from 15 to 50 . The stress is approximately 150 at the tip $(\times 10 \mathrm{MPa})$. The $\mathrm{L}$ in the center provides the scale in the two directions (ratio $1: 2)$ and the horizontal bar has for length $1.8\left(\times 10^{-8} \mathrm{~m}\right)$.

have been stopped when the formation of the dissolution pit was clear and no physical argument was proposed to assess the local failure of the material. One could think of a fracture mechanics argument based on an estimate of the stress intensity factor or invoke a plasticity yield criterion, in view of the large stresses mentioned above. For quartz tested in the lab, fracture seems to be the observed failure mechanism. However, creep plasticity is often invoked to be active in nature due to the presence of minute amount of fluid within the solid phase. Here, it suffices to say that IPS has activated a second mechanism which is contributing to irreversible modifications of the local morphology which are not captured by the present scheme.

The physical interpretation of the sudden acceleration in the dissolution rate seen in Figs. 8 and 9 is now clear. It is a localization of the phase transformation in a small portion of the block resulting in a dissolution pit or cusp. The latency period, defined by the duration of stage 1 defined above, is function of the imperfection size and the stress $\sigma_{0}$.

The role of the chemical potential on the local morphology change is now discussed. It is a constant scalar selected to have thermodynamic equilibrium pointwise along the interface for the perfect block. This value has been changed to 3.032, corresponding to an increase of $2 / 3$ of a percent with respect to the equilibrium value, to trigger a general deposition over the whole interface. The block has a length of 500, an 


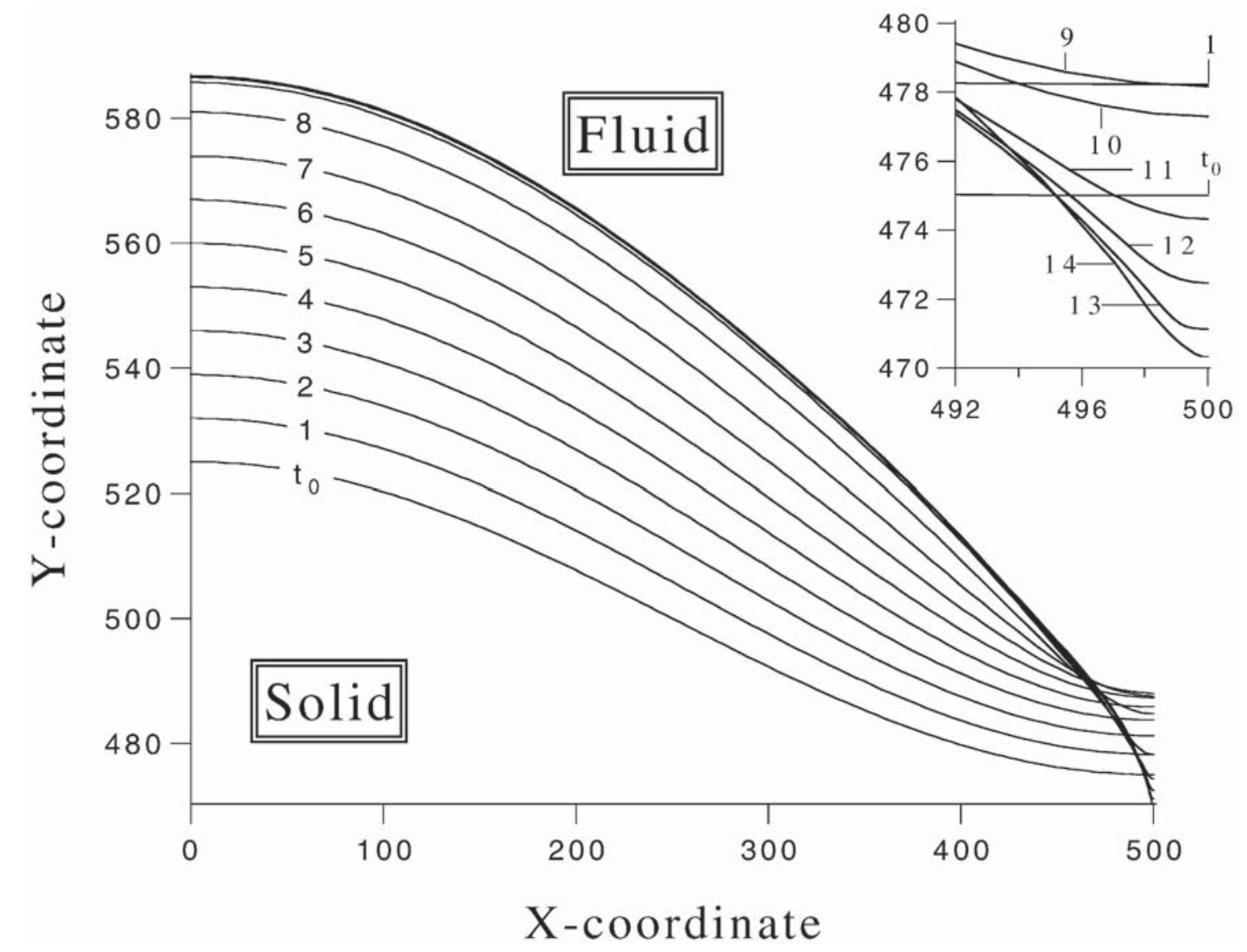

Fig. 12. Evolution of the solid-fluid interface for the square block presented in Fig. 4 and having a length of 500 and an imperfection amplitude $5 \%$ this length. The compressive stress $\sigma_{0}$ is $-18(\times 10 \mathrm{MPa})$. The chemical potential is selected to induce initially an overall deposition. However, after time $t_{7}$, the velocity at the point of largest curvature is reversed signaling dissolution through the newly added material. The various times are defined in Fig. 9 with the letter $t$.

imperfection of $5 \%$ and the stress $\sigma_{0}$ is -18 . The position of the interface at 15 times are presented in Fig. 12. These times are identified by the letter $t$ on the dotted curve of Fig. 9. The general evolution of the interface is rather similar to the one described above except for a single feature: the amplitude of the imperfection is increasing with the addition of a translation due to the general deposition. The increase in amplitude is due to the build up of a stress concentration in the thinner section of the block. This concentration is sufficient at time $t_{7}$ to balance the extra contribution of the chemical potential to the driving force and to stop the deposition process on the right-hand side of the block. From that time on, the dissolution accelerates while deposition continues on the left-hand side. This dissolution occurs through the newly added material until time $t_{11}$. After that time, the material initially present is also dissolved. Note from Fig. 9 that $t_{11}$ is already in stage 2 corresponding to the localization process. Note also from that figure that the latency period before localization is little affected by the change in chemical potential by comparing the dotted and dashed curves, the latter being obtained with the chemical potential necessary for equilibrium of a perfect block. The conclusion of this simulation is that a supersaturated pore fluid does not rule out local dissolution processes driven by stress concentrations. 


\subsection{The cylindrical tube as an open system}

The next two subsections deal with model problem 2 for a cylindrical fluid-filled cavity subjected to the loading shown in Fig. 13. The energy associated with the solid-solid interface (along the horizontal axis of symmetry) is disregarded and the thermodynamic equilibrium shape of the cavity under hydrostatic load is a circle of radius $R_{\mathrm{i}}$. This hydrostatic load results from a radial displacement $U_{\mathrm{C}}$ prescribed on the solid at a distance $R_{\mathrm{e}}$ of the center. The loading normal to the idealized grain boundary is realized by superposing to the radial displacement a vertical, negative displacement, proportional to the distance to the horizontal axis and to the positive scalar $U_{\mathrm{D}}$. This scalar is the maximum displacement magnitude prescribed and is a function of time, as shown in Fig. 13b. After a rise time $\tau_{1}$, the scalar $U_{\mathrm{D}}$ is either kept constant or brought back to zero between times $\tau_{2}$ and $\tau_{3}$. This cycle is typical of a test in the laboratory. Note that in view of the symmetry of the loading and geometry, only a quarter of the domain is studied. Material, geometrical and loading parameters are found in Table 3 if they differ from the values provided in Table 2. In this subsection the fluid phase is considered as an open system so that the chemical potential is constant in time. The case of a closed system is considered in the next subsection.

Results for problem 2, a constant chemical potential and a loading held constant after time $\tau_{1}$, are presented in Figs. 14-16. The morphological evolution of the cylindrical channel shares several features with problem 1 . This evolution allows one to distinguish two stages. First, a diffuse ovalization of the initial circular shape sets in and, second, a rapid dissolution takes place in a narrow region crossing the idealized grain boundary

(a)

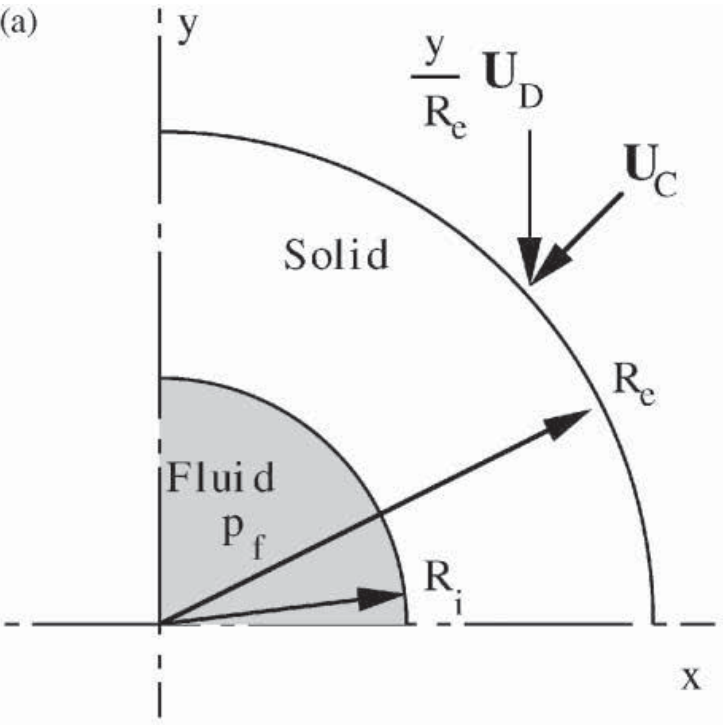

(b)

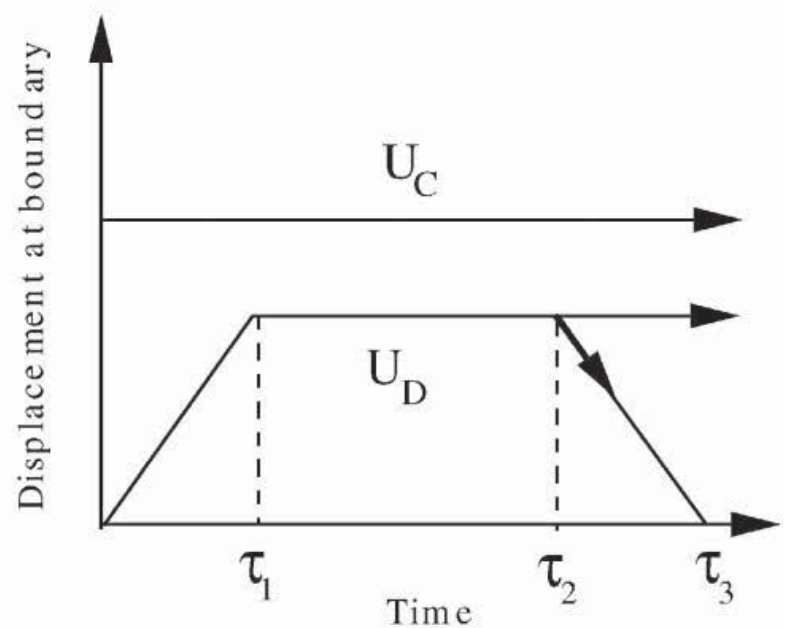

Fig. 13. Model problem 2 consists of a quarter of a cylindrical cavity with the geometry and loading shown in (a). The loading on the external radius $R_{\mathrm{e}}$ at equilibrium consists of a radial displacement $U_{\mathrm{D}}$ which remains unchanged with time. To produce a loading normal to the idealized grain boundary, a vertical displacement proportional to the vertical coordinate and having for maximum $U_{\mathrm{D}}$ is applied. The maximum value $U_{\mathrm{D}}$ has the history shown in (b) and is either kept constant after a rise time $\tau_{1}$ or brought back to zero between $\tau_{2}$ and $\tau_{3}$. 
Table 3

Physical constants, geometrical parameters and loading conditions used for the analysis of model problem 2 , if different from those presented for problem 1 in Table 2

\begin{tabular}{llll}
\hline Parameter & Unit & $\begin{array}{l}\text { Reference } \\
\text { quantity }\end{array}$ & $\begin{array}{l}\text { Dimensionless } \\
\text { value }\end{array}$ \\
\hline Constant solute chemical potential $\mu_{\mathrm{S}}$ & $\mathrm{Pa} \mathrm{m}^{3} / \mathrm{kg}$ & $\sigma_{\mathrm{R}} / \rho_{\mathrm{R}}$ & 2.997 \\
Constant scalar $R T / M_{\mathrm{S}}$ & $\mathrm{Pa} \mathrm{m}^{3} / \mathrm{kg}$ & $\sigma_{\mathrm{R}} / \rho_{\mathrm{R}}$ & 18 \\
Initial diluent phase density $\rho_{\mathrm{D}}^{0}$ & $\mathrm{~kg} / \mathrm{m}^{3}$ & $\rho_{\mathrm{R}}$ & 0.302 \\
Diluent isothermal compressibility $\kappa_{\mathrm{D}}$ & $\mathrm{Pa}^{-1}$ & $\sigma_{\mathrm{R}}^{-1}$ & $9.869 \times 10^{-3}$ \\
Initial solute concentration $C_{\mathrm{S}}^{0}$ & - & $L_{\mathrm{R}}$ & $10^{-3}$ \\
Initial internal radius $R_{\mathrm{i}}$ & $\mathrm{m}$ & $L_{\mathrm{R}}$ & 500 \\
External radius $R_{\mathrm{e}}$ & $\mathrm{m}$ & $L_{\mathrm{R}}$ & 5000 \\
Constant displacement $U_{\mathrm{C}}$ & $\mathrm{m}$ & $L_{\mathrm{R}}$ & -0.951 \\
Maximum vertical displ. $U_{\mathrm{D}}$ & $\mathrm{m}$ & $t_{\mathrm{R}}$ & -2.5 and -4.5 \\
Rise time $\tau_{1}$ & $\mathrm{~s}$ & $t_{\mathrm{R}}$ & $10-2$ \\
Beginning of unloading $\tau_{2}$ & $\mathrm{~s}$ & $t_{\mathrm{R}}$ & 6900 and 9970 \\
End of loading cycle $\tau_{3}$ & $\mathrm{~s}$ & 7200 and 10000 \\
\hline
\end{tabular}
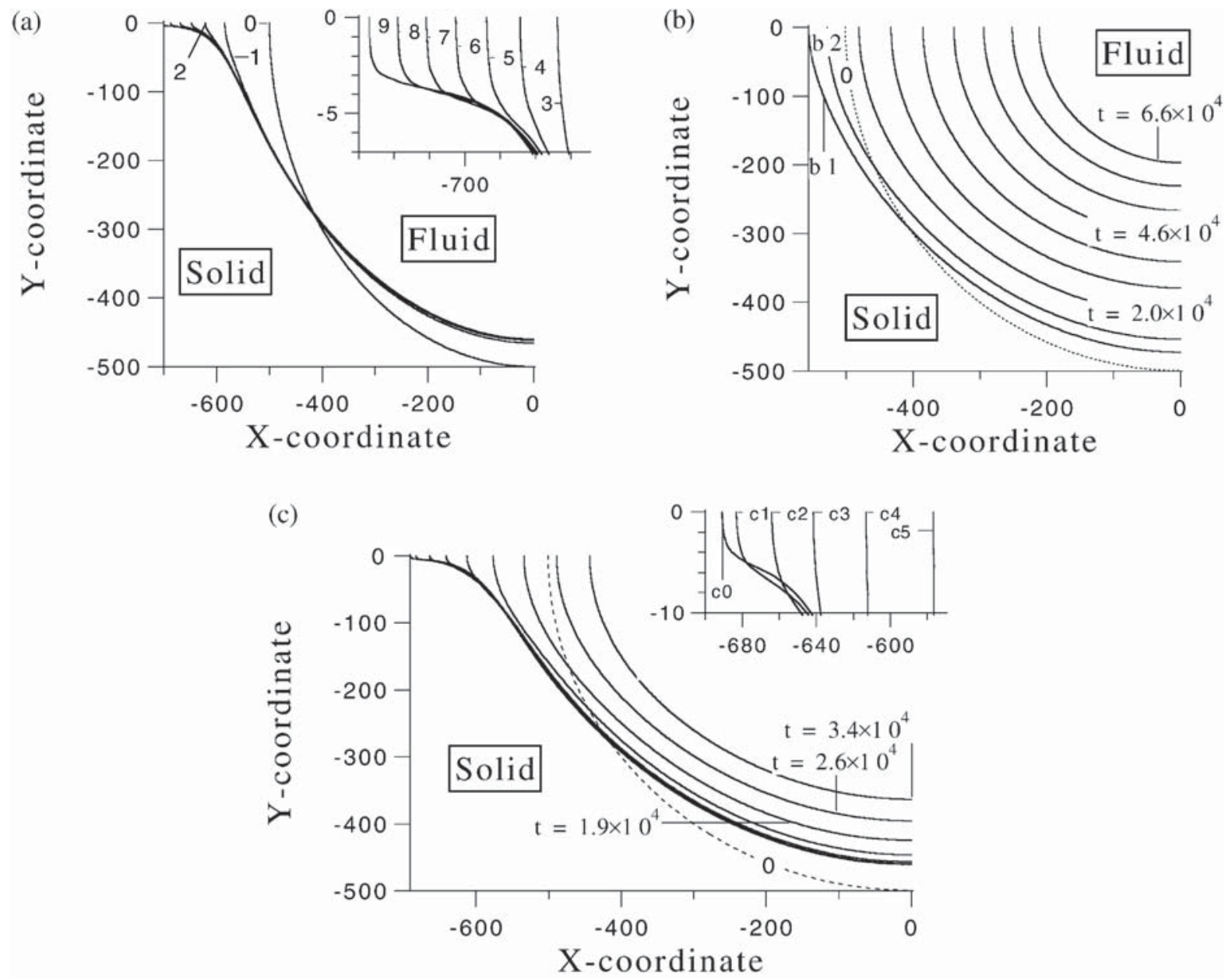

Fig. 14. Morphology evolution of the cavity, which equilibrium shape is circular, during a loading cycle normal to the idealized grain boundary (horizontal axis). In (a), the loading is held constant after a short rise time and dissolution is localized along the grain boundary. In (b) and (c), the loading is brought back to the equilibrium condition in a short time lapse. The various times are identified in Fig. 15. 


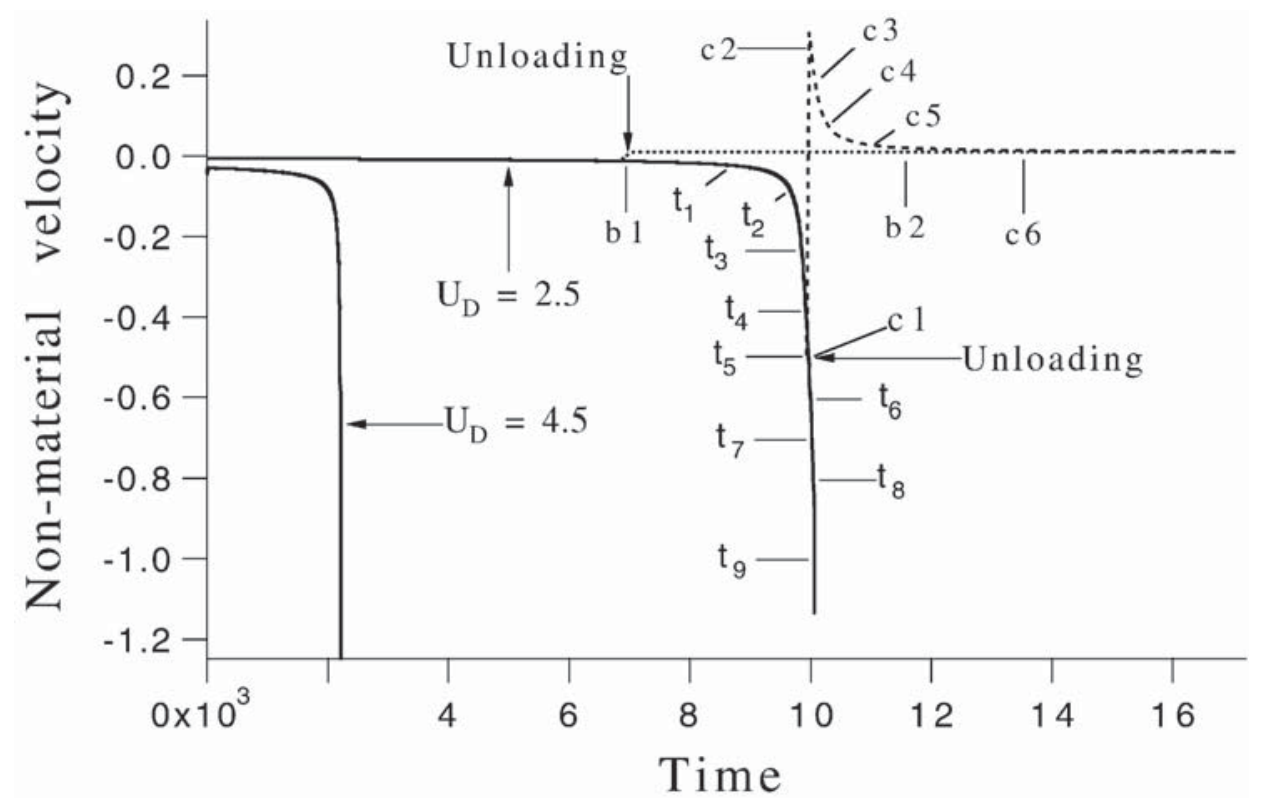

Fig. 15. Evolution with time of the non-material velocity at the point on the idealized grain boundary. The solid lines are time histories for a compressive loading normal to the grain boundary. The dotted and dashed lines are two velocity histories as the loading is brought back to the initial hydrostatic loading condition. A time of 1000 and a velocity of 1 represent $20.3 \mathrm{yr}$ and $0.5 \mu \mathrm{m} / \mathrm{yr}$, respectively.

(Fig. 14a). The position of the solid-fluid interface is plotted on that figure for 10 times defined in Fig. 15 which presents the time histories of the non-material velocity of the point of intersection between the interface and the horizontal axis (the idealized grain boundary). The velocity of localized dissolution is thus orders of magnitude larger than the initial evolution rate. This rapid dissolution takes place along the idealized grain boundary providing a mechanism for the fluid to penetrate the dry contact region. This undercutting of the dry contact or island leads to the creation of a fluid film which, at time $t_{9}$, has a thickness 4 times the characteristic length (inset of Fig. 14a). It is thus surface tension which appears to control the film thickness in our simulation. The undercutting of the contact takes place after a latency period necessary for the build up of the stress concentration. As in problem 1, this latency period is shortened by increasing the normal load, as is seen for the solid curve in Fig. 15 obtained for a $U_{\mathrm{D}}$ of 4.5. The distribution of stress in the region ahead of the dissolution front is presented in Fig. 16 in the form of 26 isocontours of equivalent Von Mises stress ranging from 1.5 to $20(\times 10 \mathrm{MPa})$ and obtained at time $t_{9}$. Note, as in problem 1 , the typical stress distribution of a fracture mechanics problem in the region ahead of the undercutting. The stress concentration observed is responsible for the large Helmholtz free energy at the tip and the increasing velocity at which the undercutting of the dry contact region takes place.

An important issue arising with the interpretation of experimental procedure is the nature and the extent of possible changes in the microstructure during unloading and prior to the drying of a grain contact necessary for the observation. To assess the importance of the duration of unloading, we have conducted two unloadings starting at time $\mathrm{b} 1$ and $\mathrm{c} 1$ defined by the intersections of the dotted and dashed curves with 


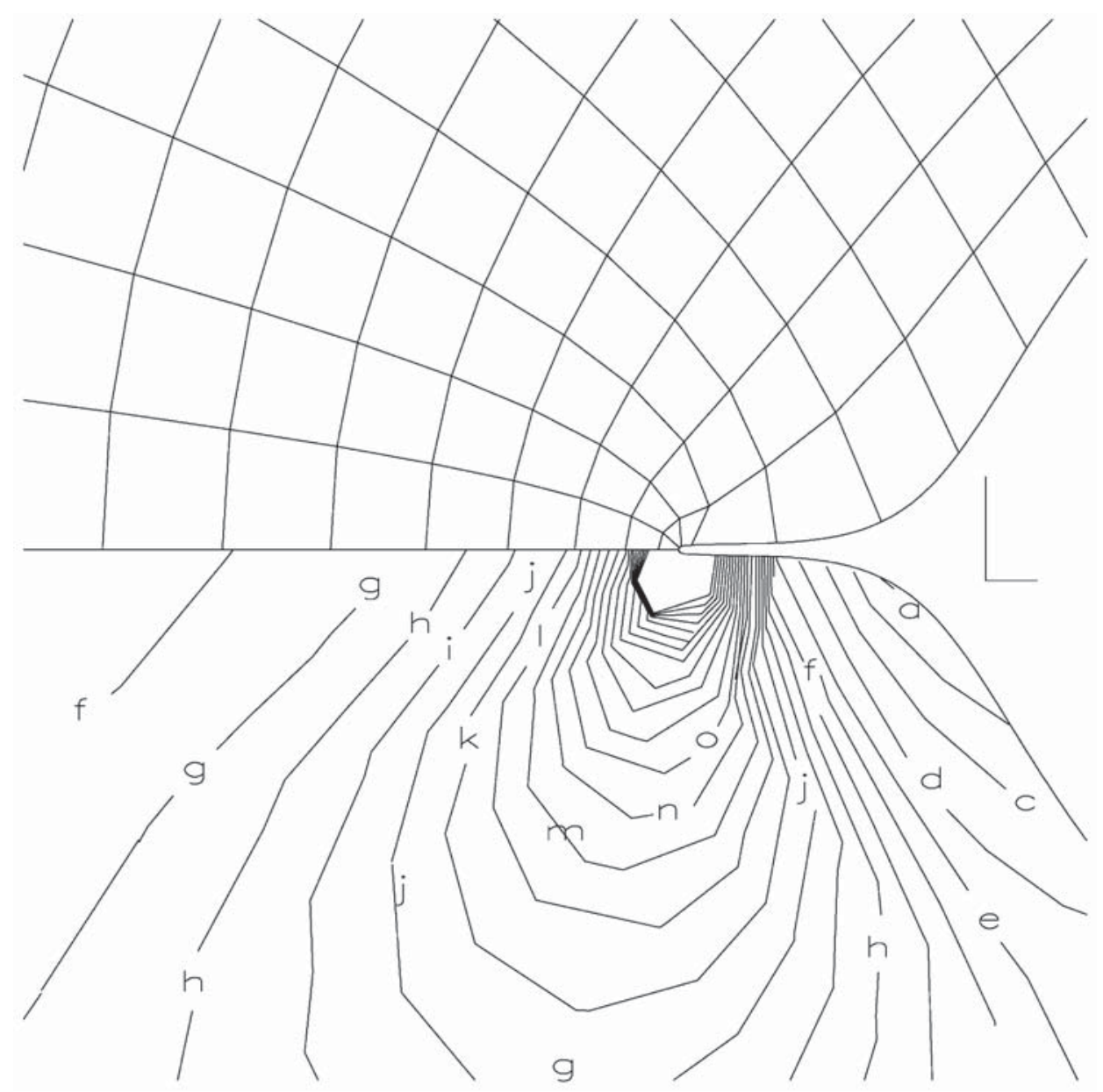

Fig. 16. The mesh and 26 isocontours of equivalent Von Mises stress in the region ahead of the localized dissolution at time $t_{9}$ defined in Fig. 15. The isocontours $(\mathrm{a}-\mathrm{z})$ range from 1.5 to $20(\times 10 \mathrm{MPa})$. The $\mathrm{L}$ on the right provides the scales in the two directions (ratio $2: 1$ ) with the horizontal bar having for length 33.3 $\left(\times 10^{-8} \mathrm{~m}\right)$.

the solid curve in Fig. 15. Unloading occurred over a period $\tau_{3}-\tau_{2}$ which is for the first and second unloading 330 times the characteristic time, respectively (see Table 3 ). The first unloading takes place before the localized dissolution (during stage 1) and the ovalization is reversed towards the equilibrium shape marked by a dotted curve in Fig. 14b. This reverse process is slow as can be judged by the times $b_{1}$ and $b_{2}$, defined in Fig. 15, at which the two profiles are plotted in Fig. 14b. Furthermore, the initial equilibrium shape is not stable, as discussed in Leroy and Heidug (1994), and the cavity keeps on closing at a very slow rate, as can be seen by the times provided in that figure. Note that the solid-fluid interface of the closing tube is not circular and retains the elliptical shape inherited during loading.

The second simulation of unloading is initiated during stage 2 at time $c_{1} \simeq t_{5}$. The localized dissolution is already well defined (see Fig. 14a). The unloading can then also be described as a two-stage process. The first stage lasts as long as the duration of the unloading and is characterized by a healing of the newly formed film due to a rapid deposition in that region. This stage is recognized in Fig. 15 by the change in 
sign of the velocity of the tip of the dissolution pit to a value which is, however, of same magnitude as the dissolution velocity. Again, this change in non-material velocity occurs during the unloading $\tau_{3}-\tau_{2}$. The second stage of the closing of the newly formed thin film occurs after time $c_{3}$ and corresponds to a rather diffuse morphology change over the whole interface. The rate at which the closing of the cavity occurs is then identical to the one found in the first simulation of unloading. Furthermore, by comparing the shapes of the solid-fluid interface in Fig. 14c, we observe that the memory of the existence of the thin film is completely lost in the long term.

The rapid closure of the newly formed film during stage 1 of unloading deserves some comments. It can be explained by looking at the expression for the driving force $\chi$ in Eq. (12) and at the isocontours of equivalent Von Mises stress prior to unloading, which are close to those displayed in Fig. 16. The Von Mises stress $\sigma$ is then of the order of 50 at the tip of the newly formed film and the Helmholtz free energy $\left(\psi_{\mathrm{s}} \simeq \sigma^{2} / E\right)$ is of order 1 , in view of the material properties given in Table 2. The Helmholtz free energy is the leading term in the driving force and this value of 1 is consistent with the non-material velocity found at time $t_{5}$ in Fig. 15 according to the kinetic law (11). Upon unloading, the stress distribution in the region of the cusp tip changes drastically. If the final loading could be described by an external pressure identical to the internal pressure, a constant state of hydrostatic stress would prevail in the solid phase for any solid-fluid interface morphology. This result is of course valid in the absence of surface tension which modifies the local normal stress vector acting on the solid-fluid interface depending on the curvature, in accord with Laplace's boundary conditions (4). To document the effect of curvature on the stress distribution, 26 isocontours of equivalent Von Mises stress are plotted in Fig. 17 at time $c_{3}$. This time is just after the peak in non-material velocity during unloading (Fig. 15). The shape of the interface has not yet been modified from time $t_{5}$ but the stress distribution is already very different from the one described above at time $t_{9}$. Surface tension results in a positive traction at the tip of the newly formed film which reduces the value of the equivalent stress compared to the inner part of the solid phase. It is now at the tip of the film that the stress magnitude is minimum (marked by a minus sign). The maximum stress is found away from the dissolution region and is marked by a plus sign in Fig. 17. Considering now the driving force for interface migration, the Helmholtz free energy at time $c_{3}$ is $1 / 1000$ the value taken at time $t_{9}$. The dominant contribution to the driving force is now due to surface tension (of order $2 H$ ). The radius of curvature being of order 1, see Figs. 16 and 17, the positive value of the non-material velocity at time $c_{3}$ of order 0.3 is thus well explained by the kinetic law (11) and the driving force (12).

\subsection{The cylindrical tube as a closed system}

The last set of results to be presented concerns problem 2 (Fig. 13) for a closed system: the compressible fluid phase is isolated within the grain boundary and the diluent mass is preserved. Diffusion is fast within the fluid which is assumed homogeneous at all times. The pore pressure and solute concentration are thus computed at every time step as described in Section 2 and in Appendix A. 


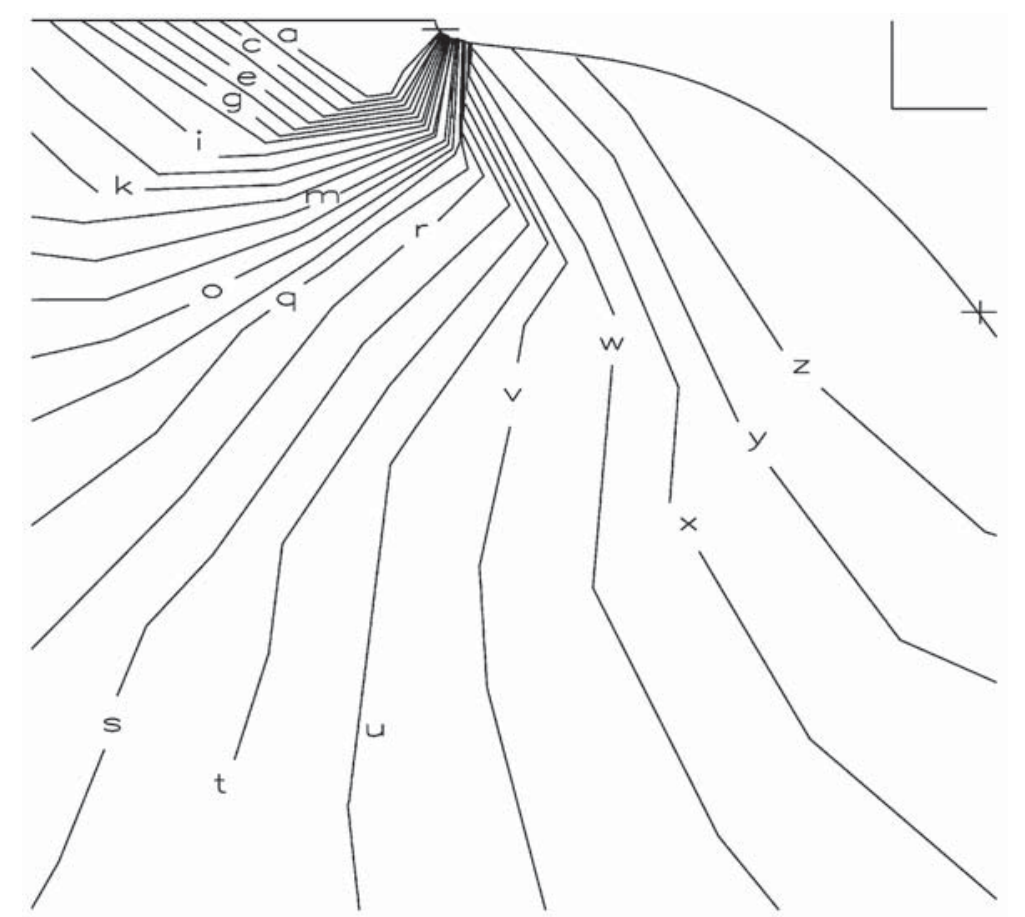

Fig. 17. Isocontours of equivalent Von Mises stress are plotted in the zone close to the grain boundary where localized dissolution took place before unloading. The 26 isocontours $(\mathrm{a}-\mathrm{z})$ range from 1.10 to 1.25 $(\times 10 \mathrm{MPa})$. Note that, due to the action of surface tension, it is not the maximum, marked by a plus sign, but the minimum, found at the position of the minus sign, which is at the grain boundary contact. The $\mathrm{L}$ in the top right corner provides the length scale: $20\left(\times 10^{-8} \mathrm{~m}\right)$.

To understand the non-linear evolution of the pressure, the solute concentration and thus of the solute chemical potential, it is first proposed to consider the model problem 2 (Fig. 13a) in which the loading is radial at all times. The radial displacement results from the superposition of a constant $U_{\mathrm{C}}$ and a variable $U_{\mathrm{D}}$ having the same history as shown in Fig. 13b. The loading being radial, the problem is axisymmetric with an analytical solution for the driving force for any value of the inner radius $R_{\mathrm{i}}$. The problem solution is nevertheless time dependent and requires the numerical scheme presented by Ghoussoub (2000). The results are presented in Fig. 18 where the fluid pressure, solute concentration, solute chemical potential and thermodynamic force are plotted as a function of time. Material, geometrical and loading parameters have the values provided in Table 3 .

The evolution of the fluid pressure presented in Fig. 18a is dominated by the compressibility of the fluid phase, since there are small differences between the solid and the dotted curve, the latter being obtained in the absence of a phase transformation (no PT). This latter curve is composed of straight segments connected at the end of the rise time $\tau_{1}$, the beginning of unloading $\tau_{2}$ and at the end of the loading cycle $\tau_{3}$. There is thus little influence on the pressure of the variation in solute concentration presented in Fig. 18b. This solute concentration decreases by 6\% during the loading cycle. Note the time delays due to the kinetic law at the beginning of the cycle and at any change in loading conditions (times $\tau_{1}, \tau_{2}$ and $\tau_{3}$ ). Another feature seen on those first two graphs of Fig. 18 is the constant state of pressure and solute concentration 

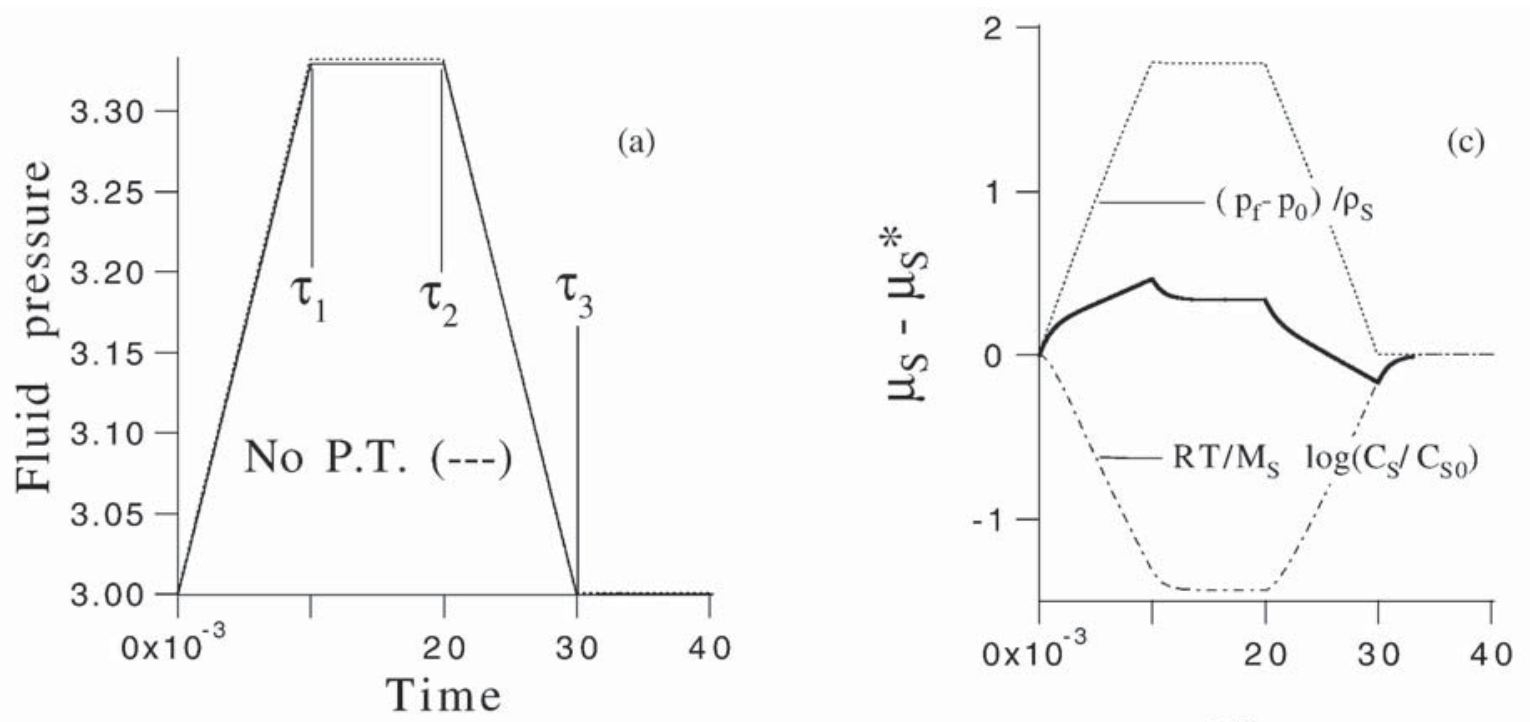

Time
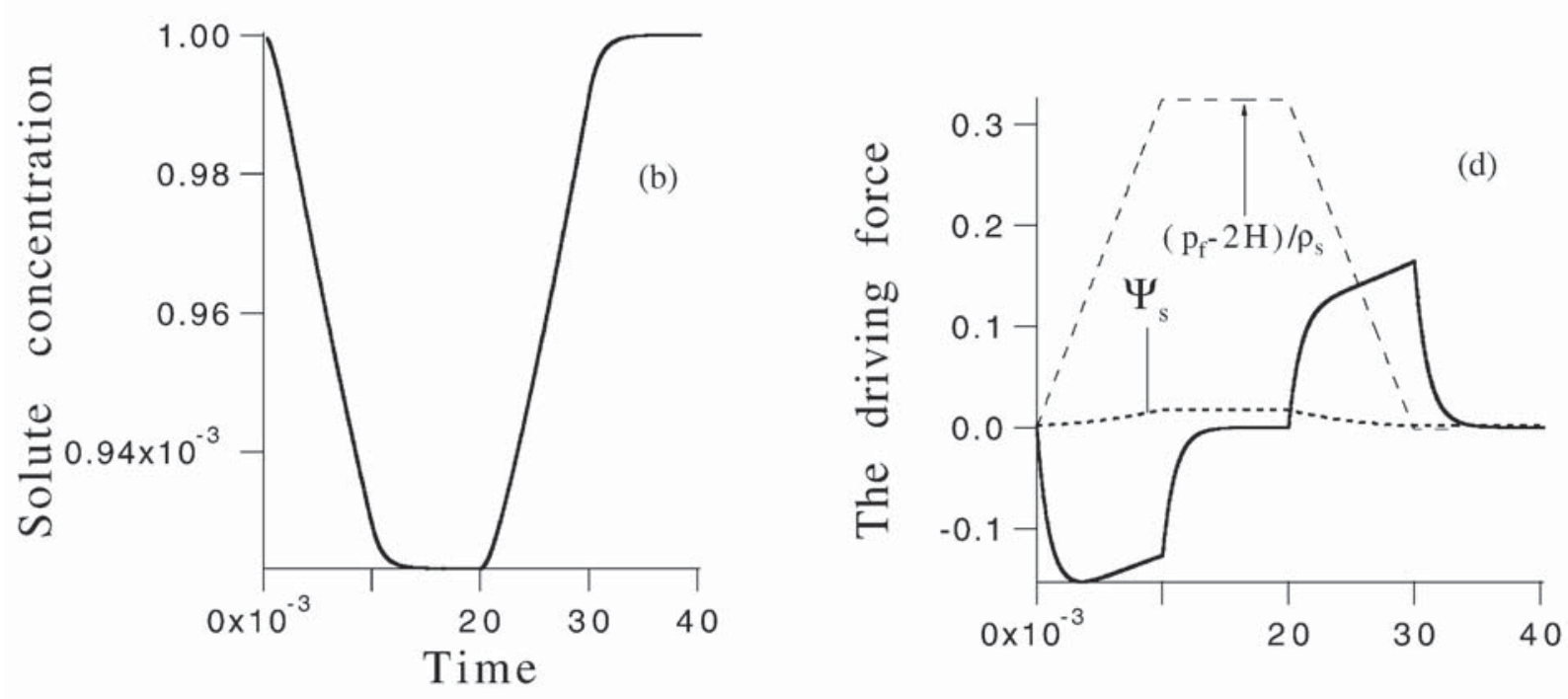

Time

Fig. 18. Evolution with time of the fluid pressure (a), the solute concentration (b), the solute chemical potential (c) and the thermodynamic force (d), during a radial loading cycle on the cylindrical cavity of Fig. 13. The radial displacement is the constant $U_{\mathrm{C}}$ plus the scalar $U_{\mathrm{D}}$, the history of which is shown in Fig. 13b.

reached before unloading: the system has achieved a new equilibrium which is stable to homothetic perturbation. Such a stable equilibrium shape was not present for open systems and is due to the mechanical interaction between the solid and fluid phases.

The next quantity studied during the radial loading of the cylindrical cavity is the chemical potential (Fig. 18c). The solid curve represents the variation in chemical potential with respect to the equilibrium value $\mu_{\mathrm{S}}^{*}$. This variation is due to two contributions according to Eq. (9), the first resulting from the pressure dependence of the chemical potential and the second from the variation in solute concentration. Those two contributions are presented by the dotted and dotted-dashed curves in Fig. 18c, respectively. The first contribution leads to an increase in the chemical potential during 
loading while the second contribution is negative and is due to the decrease in solute concentration. Note that the dotted-dashed curve exhibits the delays described above when loading is initiated or modified. It is the sum of these two contributions which results in the complex variation of the chemical potential, which increases first up to time $\tau_{1}$ (influence of $p_{\mathrm{f}}$ ) and then decreases once loading is held constant (delay due to the deferred evolution of the solute concentration). This pattern repeats itself during the second part of the loading cycle. The same complexity is found for the driving force defined in Eq. (12) and plotted in Fig. 18d as a solid curve. This evolution is dominated by the variation of the fluid pressure and the chemical potential. This conclusion is reached by comparing the solid, dotted and dashed curves in Fig. 18d corresponding to the driving force, the Helmholtz free energy, and the term $\left(p_{\mathrm{f}}-2 H\right) / \rho_{\mathrm{s}}$ which is the third on the right-hand side of the definition of the driving force (Eq. (12); note that the dimensionless $\rho_{\mathrm{s}}^{-1}$ is equal to $1+\operatorname{tr}(\varepsilon)$ ). The Helmholtz free energy constitutes a small contribution to the driving force in the absence of any stress concentration. The variation of the pressure term is as important as the variation of the solute chemical potential, as it can be seen by comparing the solid curve of Fig. 18c and the dashed curve in Fig. 18d. The difference between the latter and the former determines mostly the evolution of the driving force. Note that this driving force is close to zero during most of the interval $\tau_{3}-\tau_{2}$, expressing the proximity of an equilibrium state. The variations of the four quantities presented in Fig. 18 are now going to be used to explain the initial morphology changes of the solid-fluid interface during a loading normal to the idealized grain boundary.

The results for the evolution of the cylindrical cavity during a loading normal to the grain boundary with an isolated fluid phase are presented in Figs. 19-23. The evolution of the closed system on the time scale considered to generate the normal loading is first commented prior to the analysis of the long term evolution. The initial evolution of the pressure, the solute concentration, solute chemical potential and the maximum non-material velocity are presented in the insets of Figs. 19 and 21. The application of a displacement normal to the grain boundary, on top of the radial loading constant in time, results in a compression of the fluid phase and an increase in the fluid pressure which is controlled by the compressibility of these two phases as for the radial loading discussed above (compare Fig. 18a and inset of Fig. 19a in which the solution in the absence of any phase transformation is indicated by a dashed curve). The evolution of the solute concentration is also similar to the one discussed previously since it decreases by $4 \%$ until the rise time $\tau_{1}$ is reached and tends to a plateau afterwards, inset of Fig. 19b. The solute chemical potential evolves in time with the pressure and the concentration in accord with the uniform radial analysis (see inset of Fig. 19c): the chemical potential increases by 0.4 before decreasing towards a plateau once the rise time $\tau_{1}$ is reached. The value of the driving force cannot be presented in a way similar to $\mu_{\mathrm{S}}$ since the loading results in a heterogeneous stress state around the cavity. We can nevertheless analyze the non-material velocity of the interface at the intersection of the idealized grain boundary, inset of Fig. 21. There, the velocity is positive, increasing from time zero to $\tau_{1}$, and decreasing afterwards as if an equilibrium state was acting as an attractor. This positive velocity signals a deposition as for a uniform radial loading. 

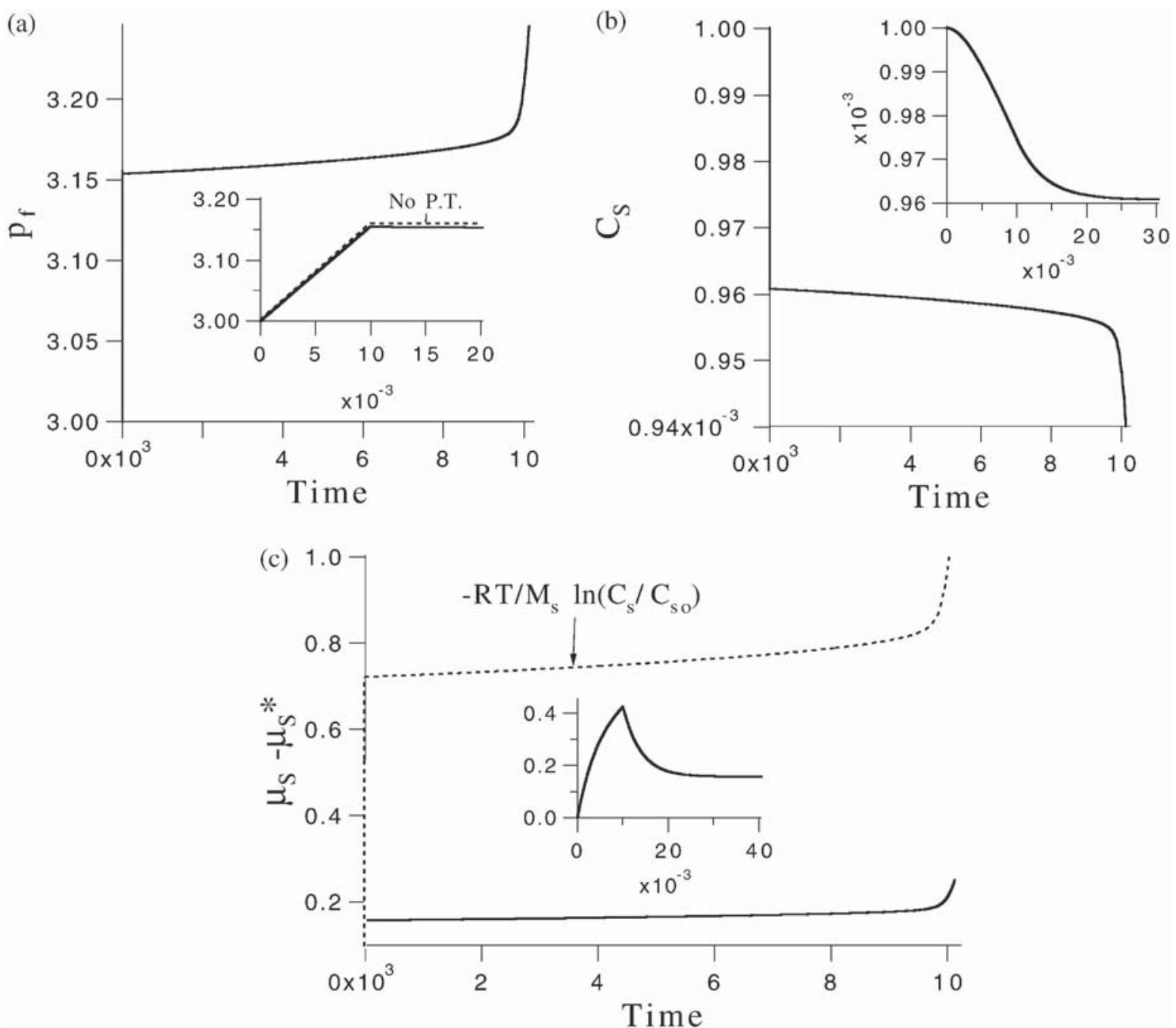

Fig. 19. Evolution with time of the fluid pressure (a), the solute concentration (b), and the variation of the solute chemical potential (c) during the loading of the cylindrical cavity shown in Fig. 13. The fluid phase is assumed isolated. In insets, the evolution of the three quantities of interest is shown on the time scale of the rise time.

The heterogeneous stress state generated by the loading normal to the grain boundary makes itself felt mostly in the long term. However, its effects can already be seen on the time scale of $\tau_{1}$ from the distribution of non-material velocities over the interface presented in Fig. 20 at times $0.77,2.30$ and 2.45 times $\tau_{1}$. The vectors in that figure are scaled with respect to the maximum magnitude in velocity which are $0.25,1.3 \times 10^{-2}$ and $6.6 \times 10^{-3}$ at the 3 times denoted (a), (b) and (c) and defined in the inset of Fig. 21. The distribution in Fig. 20a shows the general deposition over the whole interface during the application of the loading normal to the grain boundary. The consequence of the stress heterogeneity on the non-material velocity is then not important. Once this loading is established, the non-material velocity decreases and the stress gradient manifests itself by a smaller non-material velocity close to the idealized grain boundary (Fig. 20b). At time $2.45 \tau_{1}$ (Fig. 20c), this effect is sufficiently important for the velocity in the region of the grain boundary to be reversed and for the newly added material to start to dissolve. From that time onwards, the evolution of the closed system resembles 


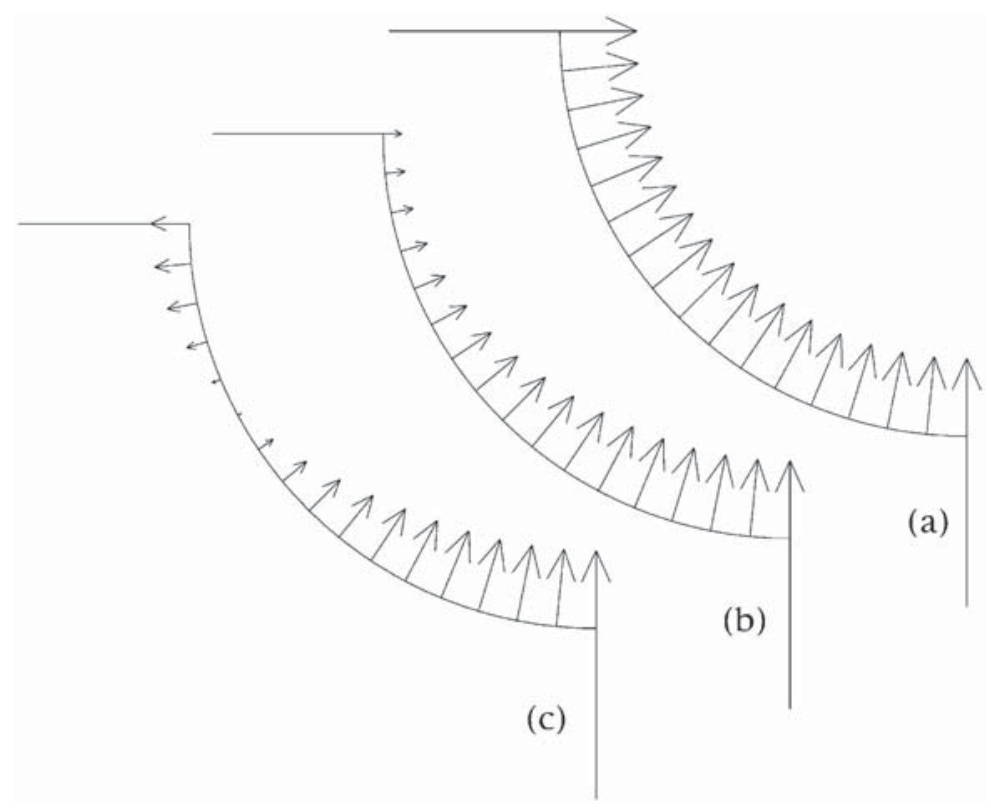

Fig. 20. The non-material velocity on the solid-fluid interface at three different times, $0.77,2.30$ and 2.45 times the rise time $\tau_{1}$ in (a), (b) and (c), respectively. The size of the vectors are normalized by the maximum velocity magnitude which is $0.25,1.3 \times 10^{-2}$ and $6.6 \times 10^{-3}$ at the three times.

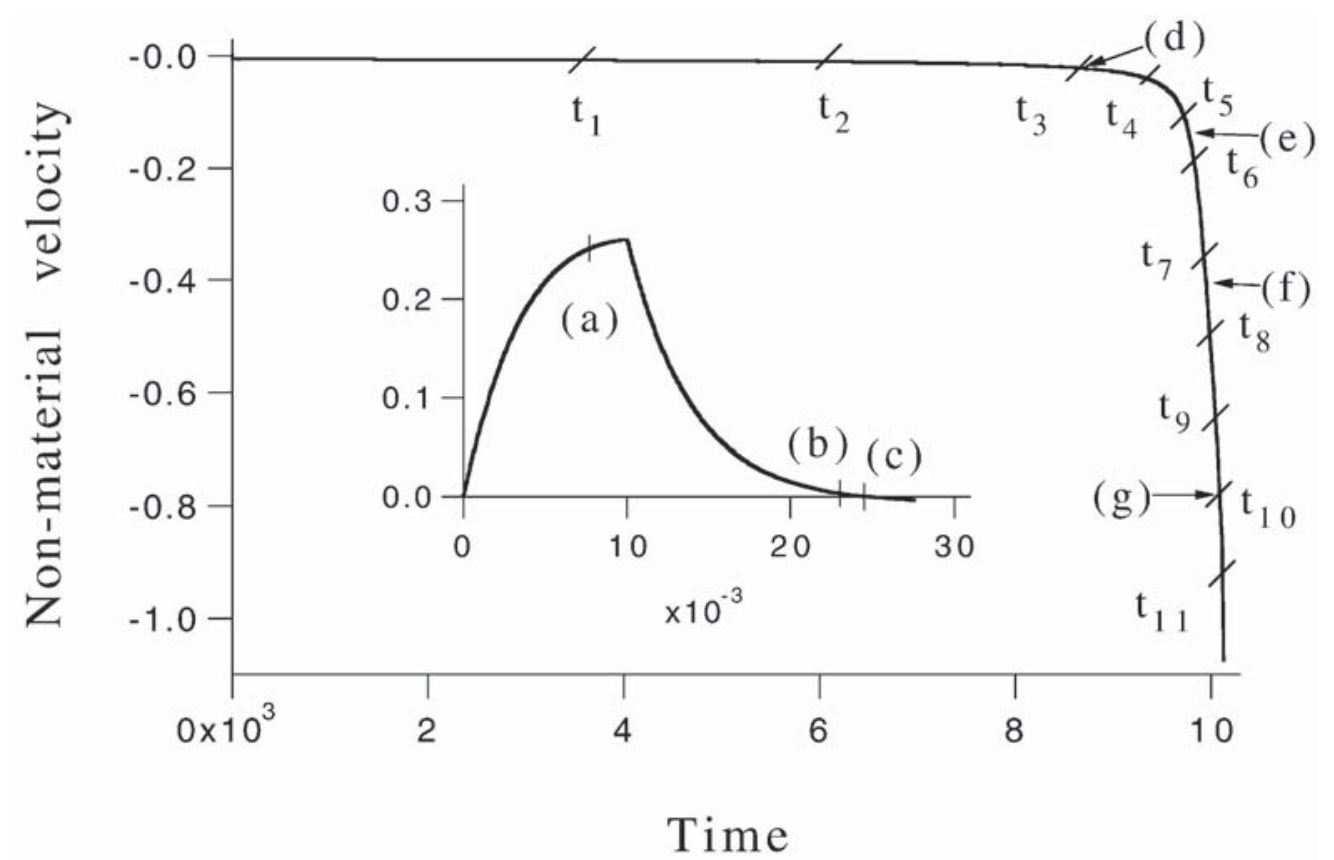

Fig. 21. History of the non-material velocity at the point of the solid-fluid interface in contact with the idealized grain boundary. In the inset, this history is presented on the time scale of the rise time.

more the one described in the previous section for an open system except for a major difference which is discussed next.

The second phase of the morphology evolution occurs on a very different time scale which is 6 orders of magnitude larger than the rise time. This is the time scale necessary for the ovalization of the cylindrical cavity to be sufficient to generate a stress concen- 


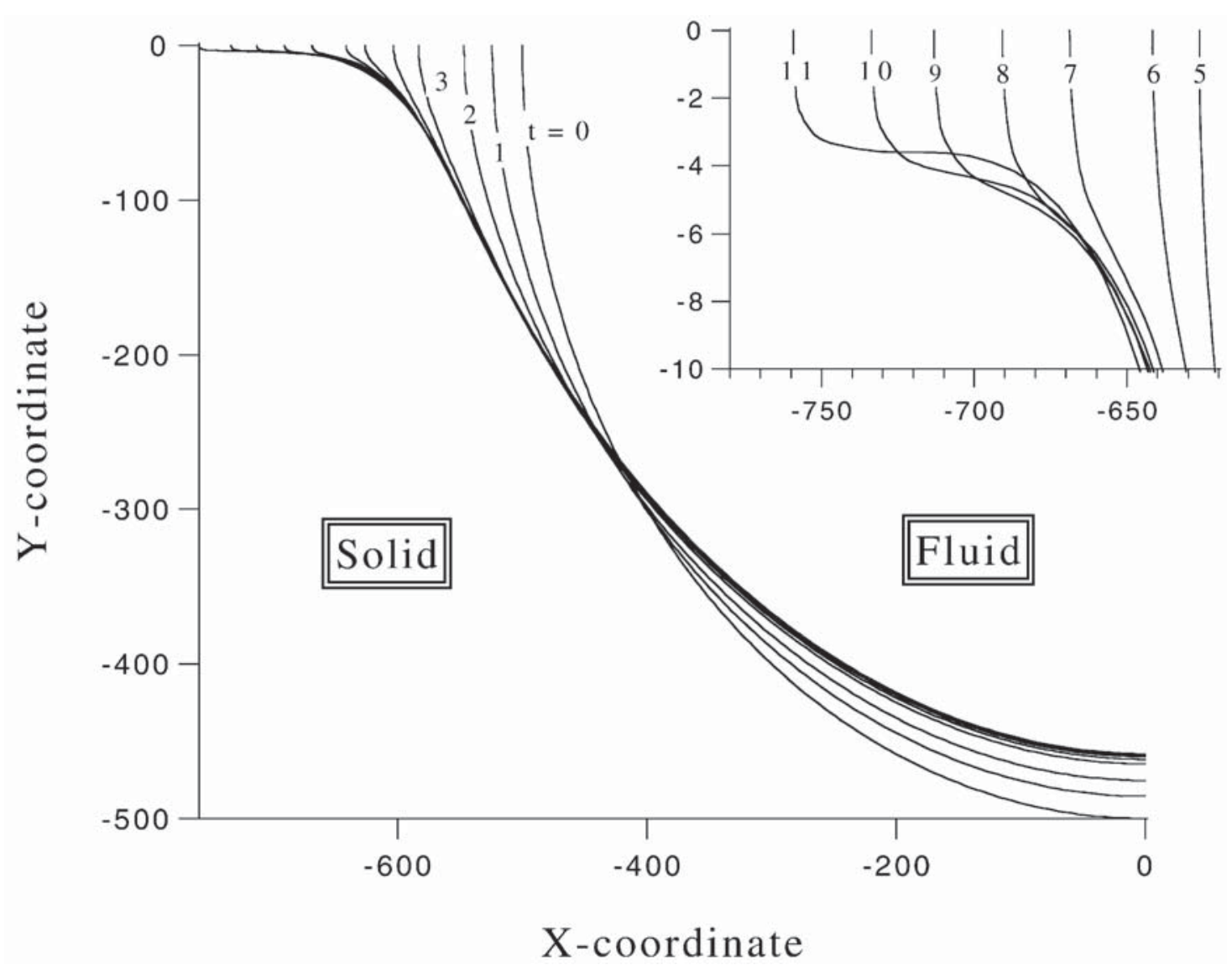

Fig. 22. The position of the interface for the problem of a cylindrical cavity of initially circular cross section and for a closed system, at 12 times defined on the previous figure.

tration along the idealized grain boundary. This ovalization and the consequence of the stress concentration build up can be seen in Fig. 22 for 12 times which are defined in Fig. 21. This long term evolution is thus similar to the one discussed in the previous section: once the stress concentration is established after a latency period, there is a rapid dissolution process which provides a mechanism for the fluid to wet the grain boundary and to create a fluid film. The velocity of the tip of the newly formed thin film is then several orders of magnitude larger than during the latency period. The difference between the undercutting mechanism along the idealized grain boundary for closed and open systems can now be judged by comparing the insets of Figs. 22 and 14a. It can be seen that for the closed system there is a deposition along the newly formed film over the $X$-coordinate -650 to -750 . To understand this thinning of the film, one has first to recognize that the solute concentration in the fluid phase keeps on decreasing during the undercutting, (Fig. 19b). The rate of decrease correlates with the increase in tip non-material velocity. The pressure is also found to increase at a similar rate with the tip non-material velocity. This build up in pressure indicates that the volume occupied by the fluid phase is decreasing during the wetting of the grain boundary. There is thus more material deposited over the interface than dissolved at the tip of the undercutting zone. The combination of the evolution of the fluid pressure and the solute concentration leads to an increase in the chemical potential in time 


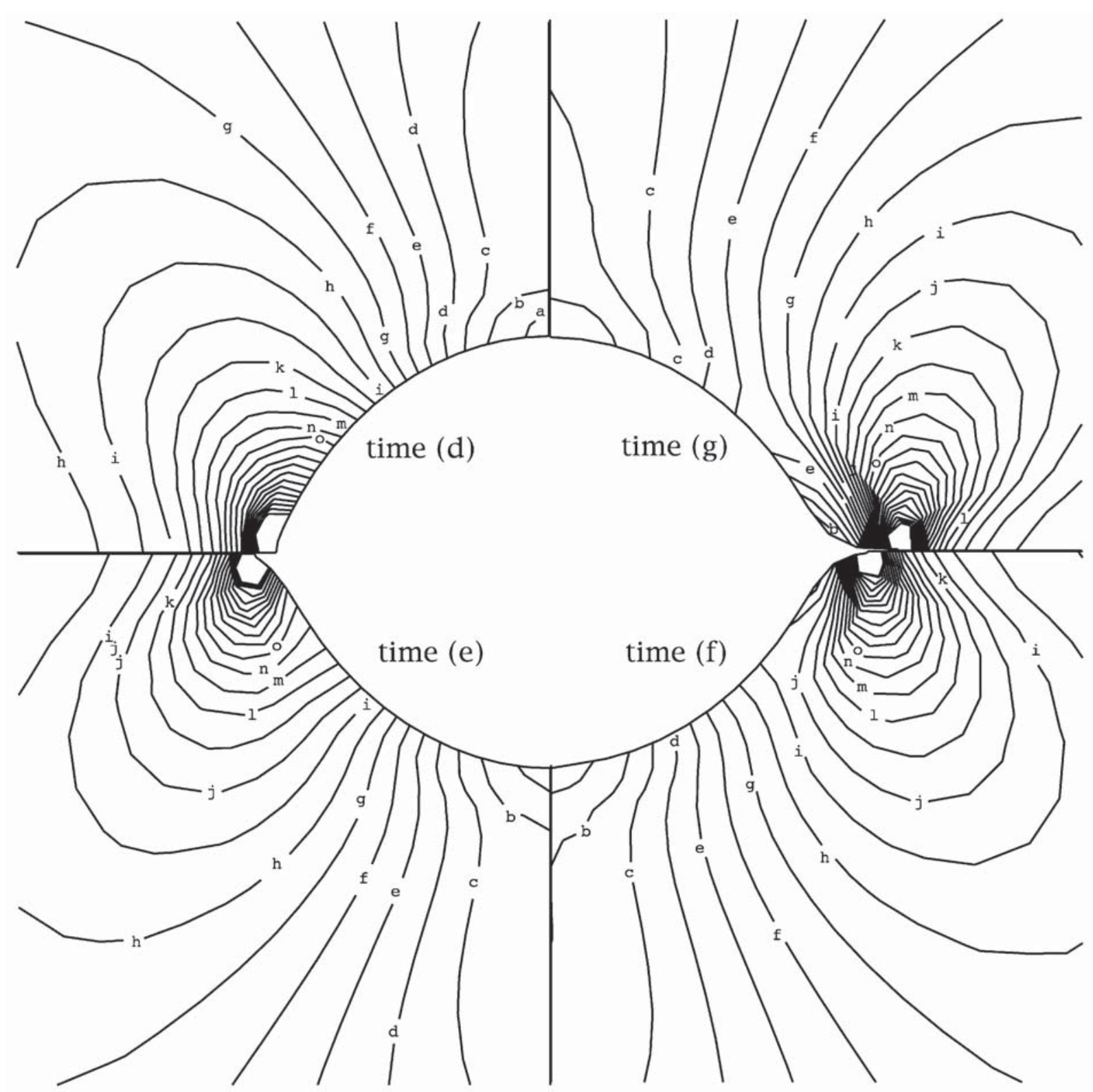

Fig. 23. 26 isocontours of equivalent Von Mises stress ranging from 1.5 to $12(\times 10 \mathrm{MPa})$ at four times labeled (d) $-(\mathrm{g})$ and defined in Fig. 21.

(Fig. 19c). To understand the consequences of this increase which results in a change of sign of the driving force over the solid-fluid interface the various contributions to the driving force should be documented as in Ghoussoub (2000).

The conclusion of such a detailed analysis can be comprehended by studying the distribution of equivalent Von Mises stress at 4 times labeled (d)-(g) and defined in Fig. 21. Fig. 23 presents 26 isocontours at each time in a quarter of the figure, starting with time (d) in the upper right corner and counting anticlockwise. The isocontours range always from 1.5 to $20(\times 10 \mathrm{MPa})$ for sake of comparison. It can be seen that, as the undercutting develops, the crack-like behavior results in a general unloading away from the tip. For example, the isocontour $i$ which corresponds to a stress of five intersects the cavity at a point which is shifted towards the undercutting tip as times 
changes from (d) to (f). At time ( $g$ ), this level of stress is not found any more on the solid-fluid interface within the cavity. The evolution of the stress concentration towards a crack-like stress distribution is thus responsible for the change in sign of the driving force over most of the cavity wall and, consequently, for the thinning of the newly formed film.

\section{Conclusion}

This paper has given some insight on the solid-fluid phase transformation which takes place within grain boundaries of porous rocks compacting by the deformation mechanism IPS. For that purpose, two initial- and boundary-value problems have been proposed, motivated by the channel and island structure observed within grain boundaries after experiments (Urai et al., 1986; Spiers et al., 1990).

The assumptions common to the two problems are that the phase transformation is controlled by a linear kinetic law which relates the mass flux to the difference between the chemical potential of the solid phase at the interface and of the solute in the fluid phase. The solid is isotropic, linear elastic and diffusion occurs only within the fluid phase assumed homogeneous and in chemical and mechanical equilibrium at all times. The numerical method relies on an explicit scheme and is based on the finite-element method. At every time step, the state of stress is determined over the domain occupied by the solid phase and the non-material velocities of the phase transformation are computed over the solid-fluid interface, thanks to a weak formulation of the kinetic law. The boundary of the solid phase is then migrated based on that velocity distribution prior to an adaptation of the finite-element mesh over the solid phase. The feature of the family of finite elements proposed is a $C_{1}$ continuity along the interface and a $C_{0}$ continuity in the bulk obtained by combining Hermite's and Lagrange's polynomials. This enhanced continuity on the interface was found necessary to capture properly the influence of surface tension.

The first of the two problems is motivated by the possible existence of fluid films within grain boundaries. The solid phase has initially the shape of a rectangular block and is in contact on one of its side with the fluid phase of the film. The stability of the flat interface is studied first by a perturbation analysis which reveals that the difference between the interface parallel compressive stress and the fluid pressure is destabilizing. Surface tension, on the contrary, is stabilizing. The combination of the two opposing factors results in the selection of a dominant perturbation with a specific wavelength having the fastest rate of growth. There are two interesting outcomes from this linear stability analysis conducted on a domain of finite dimensions. The first is that the limit for an infinitely long wavelength does not correspond to the stability predictions obtained for a homothetic mode. This is due to the presence of a singularity in the stability boundary conditions. This result could explain similar singular limits found in bifurcation and stability analyses such as the one conducted by Leroy and Molinari (1993) to detect patterns in shear zones. The second advantage of this linear stability analysis is the validation of the numerical scheme by comparing analytical predictions and numerical results during the initial development of the perturbations. The long term 
evolution of the trigonometric perturbation obtained by numerical means is described as a two-stage process. During stage 1, the morphology evolution is diffuse over the whole interface: the amplitude of the trigonometric perturbation increases with time if the interface is unstable. Stage 2 is characterized by the formation of a cusp or dissolution pit in the region where the initial perturbation resulted first in an increase in the solid Helmholtz free energy. The curvature at the tip of the cusp is related to the characteristic length due to surface tension at the end of the simulation. However, the rate of dissolution keeps on increasing at all times revealing the crack-like behavior at the tip of the cusp. The latency period, defined by the duration of stage 1, is observed to decrease with an increase in the magnitude of the initial compressive stress and in the initial amplitude of the perturbation. It was also shown that a supersaturated fluid in contact with the solid does not prevent such localized dissolution to occur if the stress concentration is sufficient.

The geometry of the second problem is a cross section through a typical channel observed within the grain boundary. The equilibrium cross section is circular since the excess energy associated to the solid-solid interface is disregarded. The compression which occurs typically during the experiments is modeled by prescribing a loading normal to the idealized grain boundary. This loading is first studied for an open system typical of a fluid phase in contact with the remote pore fluid. In that instance, the solute chemical potential and the fluid pressure are assumed constant in time. The evolution of the circular cavity occurs, as in problem 1, in two phases. There is first an ovalization of the circular cross section at a very slow rate. Once the stress concentration is sufficient along the idealized grain boundary, a rapid dissolution occurs in that region. This dissolution provides a mechanism for the fluid to penetrate the whole grain boundary by undercutting the dry contact regions and thus to create a new fluid film. The thickness of this film is governed by the curvature at the tip of the undercutting region which is of the same order of magnitude as the characteristic length of the problem due to surface tension. If the loading cycle is terminated by bringing back to zero the normal load, the evolution of the cavity is then also described in two stages. In the first stage, the newly formed film closes at a rapid rate of same magnitude as the undercutting rate. This fast rate of healing is due to surface tension which enters the driving force for phase transformation. Then, in the second stage, the cavity retains an elliptical shape and closes down in a homothetic way at a very slow rate compared to the first stage. The cavity analysis is repeated for a closed system characteristic of a fluid phase isolated within the grain boundary. The solute concentration and the fluid pressure are then two of the problem unknowns. The evolution of the cylindrical cavity as a closed system is similar to the one described for an open system except for a major difference: the stress concentration at the tip of the undercutting region results in a general decrease in the stress magnitude around the cavity. This variation in stress leads to a change in sign of the driving force away from the tip. Consequently, there is deposition over the whole domain except in the tip region, the solute concentration drops, the volume of the cavity decreases and the fluid pressure increases. Moreover, the thickness of the newly formed film decreases in time.

The relevance of these numerical results for a better understanding of the action of IPS within a grain boundary should now be discussed. It should be stressed that the 
wavy perturbations of problem 1 are observed experimentally on K-alum salt in the laboratory (den Brok and Morel, 2000). Furthermore, the cusps observed in problem 1 are reminiscent of the observations made within the grain boundary of rocks which deformed by IPS in nature (Welton, 1984). The long latency period prior to the localized dissolution, obtained with a data set typical of quartz grains at $200^{\circ} \mathrm{C}$, deserves some comments. During that period the grain boundary is partly dry and thus IPS inactive. This long period is in line with the difficulty to produce IPS with quartz in the laboratory. A better quantitative assessment of the latency period prior to the action of IPS would require a characterization of the grain boundary roughness to estimate the relevant stress concentration factor. Once this stress concentration is sufficient, we observe a fast undercutting of the dry regions. It seems that our numerical results are the first proof that a fluid can wet a dry contact region by marginal dissolution resulting in the formation of a new fluid film. If loading is interrupted, the newly formed films close partly during the lapse of the unloading. Consequently, part of the structure operating during IPS is likely to be lost when the physical observation is conducted after the experiment. There are, however, no contradictions between the observation of a channel and island structure after the experiment and the presence of thin films during the same experiment. Furthermore, extrapolating the stability analysis of our first problem to the thin films created in problem 2, it appears that this thin film is not stable if the interface parallel stress differs from the pore pressure. One should thus expect the thin film morphology to evolve toward a corrugated surface which could end up with the creation of new solid to solid contacts. This thinning of the film is found here to be enhanced in closed systems. This possible continuous reorganization in time of the grain boundary points to the direction of a dynamic internal structure within the grain boundary which has been often invoked in the literature.

\section{Acknowledgements}

Many of the ideas presented here are the fruits of numerous discussions with W.K. Heidug (Shell Research, RTS, The Netherlands), F.K. Lehner (University of Salzburg, Austria) and the researchers of the HPT Laboratory (Utrecht University, The Netherlands). The first developments of the numerical algorithm for pressure solution were realized with L. Bodin (University of Metz, France) and P. Onck (T.U. Delft, The Netherlands) during their visit to Shell Research. J. Ghoussoub has benefited from a fellowship of Ecole Nationale des Ponts et Chaussées during her doctorate studies and the project was partly financed by a grant from the French Group of Research (Geomechanics of Deep Rocks) from 1995 to 1998. This support is gratefully acknowledged.

\section{Appendix A. The constraint for a closed system}

The objective of this appendix is to derive the constraint between the volume occupied by the fluid phase, the solute mass and the fluid pressure in a closed system with a fixed diluent mass. 
The solution is dilute and the solute assumed to behave as an ideal gas sustaining the pressure $p_{\mathrm{f}}$. Consequently, the volume occupied by the solute is

$$
V_{\mathrm{S}}=\frac{m_{\mathrm{S}}}{p_{\mathrm{f}}} \frac{p_{\mathrm{f}}^{0} V_{\mathrm{S}}^{0}}{m_{\mathrm{S}}^{0}},
$$

from a reference state characterized by the pressure $p_{\mathrm{f}}^{0}$, the volume $V_{\mathrm{S}}^{0}$ and the mass $m_{\mathrm{S}}^{0}$. The diluent, say water, is assumed to have a constant isothermal compressibility $\kappa_{\mathrm{D}}$ such that the volume it occupies reads

$$
V_{\mathrm{D}}=V_{\mathrm{D}}^{0} \exp \left(-\left(p_{\mathrm{f}}-p_{\mathrm{f}}^{0}\right) \kappa_{\mathrm{D}}\right)
$$

for a reference volume $V_{\mathrm{D}}^{0}$. The total volume occupied by the fluid phase is the sum of the solute (A.1) and the diluent (A.2) volume as for an ideal mixture and is presented in Eq. (10) in the main text. It is a function of the pore fluid pressure and solute mass.

The evolution of the solute chemical potential in a system constrained by a constant mass of diluent requires the knowledge of the pore pressure and the solute concentration. The current solute mass and the cavity volume are found by integrating in time

$$
\dot{m}_{\mathrm{S}}=-\int_{\partial \Omega_{0}^{\mathrm{I}}} S_{N} \rho_{\mathrm{S}} \mathrm{d} S \quad \text { and } \quad \dot{V}_{\mathrm{S}}=-\int_{\partial \Omega_{0}^{\mathrm{I}}} S_{N} \mathrm{~d} S,
$$

prior to the computation of the solute density $\left(\rho_{\mathrm{S}}=m_{\mathrm{S}} / V_{\mathrm{S}}\right)$ and concentration $\left(C_{\mathrm{S}}=\right.$ $\left.m_{\mathrm{S}} /\left(m_{\mathrm{S}}+m_{\mathrm{D}}^{0}\right)\right)$. The fluid pressure is then found by solving Eq. (10) and the solute chemical potential from Eq. (9).

\section{Appendix B. A class of finite elements for IPS}

In this appendix one defines the shape functions for the class of elements employed in the numerical simulations. The convergence property of the elements is also briefly analyzed.

The shape functions for the basic four-noded element (plus two tangents) having a $C_{1}$ continuity along the side with $\xi_{2}$ equal to +1 result from the following products of Lagrange and Hermite polynomials:

$$
\begin{aligned}
& N_{1}\left(\xi_{1}, \xi_{2}\right)=\frac{1}{4}\left(\xi_{1}-1\right)\left(\xi_{2}-1\right), \quad N_{2}\left(\xi_{1}, \xi_{2}\right)=-\frac{1}{4}\left(\xi_{1}+1\right)\left(\xi_{2}-1\right), \\
& N_{3}\left(\xi_{1}, \xi_{2}\right)=\frac{1}{2} H_{2}\left(\xi_{1}\right)\left(\xi_{2}+1\right), \quad N_{4}\left(\xi_{1}, \xi_{2}\right)=\frac{1}{2} H_{1}\left(\xi_{1}\right)\left(\xi_{2}+1\right), \\
& N_{5}\left(\xi_{1}, \xi_{2}\right)=\frac{1}{2} H_{4}\left(\xi_{1}\right)\left(\xi_{2}+1\right), \quad N_{6}\left(\xi_{1}, \xi_{2}\right)=\frac{1}{2} H_{3}\left(\xi_{1}\right)\left(\xi_{2}+1\right)
\end{aligned}
$$

with

$$
\begin{aligned}
& H_{1}\left(\xi_{1}\right)=\frac{1}{4}\left(\xi_{1}^{3}-3 \xi_{1}+2\right), \quad H_{2}\left(\xi_{1}\right)=-\frac{1}{4}\left(\xi_{1}^{3}-3 \xi_{1}-2\right), \\
& H_{3}\left(\xi_{1}\right)=\frac{1}{4}\left(\xi_{1}^{3}-\xi_{1}^{2}-\xi_{1}+1\right), \quad H_{4}\left(\xi_{1}\right)=\frac{1}{4}\left(\xi_{1}^{3}+\xi_{1}^{2}-\xi_{1}-1\right),
\end{aligned}
$$

where the local numbering is defined in Fig. 1. Additional nodes (7-10) can be included with a construction of their shape functions and modifications of the ones presented in 
Eq. (B.1) following the procedure used for Lagrange elements in the literature (e.g., Hughes, 1987).

The family of isoparametric and compatible elements described above is complete. A uniform gradient over the element is inferred by nodal values calculated from any constant gradient field (Ghoussoub, 2000). These conditions are sufficient to ensure the convergence of this class of finite elements for elliptic problems including the mechanical equilibrium (13) and the mesh adaptation problems.

\section{Appendix C. Linear stability of the rectangular block}

This appendix provides a summary of the derivation of the stability exponent $\lambda_{\xi}$, found in the main text as Eq. (24), which characterizes the initial time evolution of trigonometric perturbations added to the otherwise flat solid-fluid interface of the rectangular block presented in Fig. 4. Details of the analysis are found in Ghoussoub (2000). This linear analysis differs from the ones published so far by the account of the finite size of the domain. Results in the long-wavelength limit are different from those obtained for homothetic growth (the geometry then remains rectangular) due to a singularity which is discussed. The analysis of this singular limit could shed light on other stability and bifurcation problems having similar limits (Leroy and Molinari, 1993 ) in view of the common structure of the analyses.

The starting point of the linear stability analysis is the proposition that the interface position is modified by an infinitesimal amount according to the real part of

$$
H(x, t)=H+\varepsilon \exp \left(\mathrm{i} \xi x+\lambda_{\xi} t\right) \quad \text { with } \varepsilon \ll 1,
$$

in which $H, \mathrm{i}$ and $\xi$ are the thickness of the rectangular block at equilibrium, the pure imaginary number and the wavenumber of the perturbation, respectively. If the solution of the linearized boundary-value problem proves that the real part of $\lambda_{\xi}$ is negative for all admissible $\xi$, then the solid-fluid interface is stable. A positive real part signals the onset of instability at the wavenumber $\xi$.

The infinitesimal change in the position of the interface results in any field variable $A(x, y)$ to be perturbed by a function $\varepsilon \delta A(x, y)$ which is decomposed in normal mode as in Eq. (C.1). The normalized function $\delta A(x, y)$ takes the form

$$
\delta A(x, y)=\hat{A}_{\xi}(y) \exp \left(\mathrm{i} \xi x+\lambda_{\xi} t\right),
$$

in terms of the unknown $\hat{A}_{\xi}(y)$, a function of the $y$-coordinate only.

The field equilibrium equations over the block of perturbed geometry are satisfied by the introduction of the Airy stress function $\phi(x, y)$. Compatibility equations are then found to require the Laplacian of $\phi(x, y)$ to equal zero which dictates that the function $\hat{\phi}_{\xi}(y)$ has the following structure:

$$
\hat{\phi}_{\xi}(y)=\left(a_{0}+a_{1} y\right) \cosh (\xi y)+\left(a_{2}+a_{3} y\right) \sinh (\xi y),
$$

in which $a_{\alpha}(\alpha=0, \ldots, 3)$ are four complex constants.

These four constants are determined by the boundary conditions over the 4 sides of the rectangular block shown in Fig. 4. Along the sides $\mathrm{AA}^{\prime}$ and $\mathrm{AB}$, the zero 
normal displacement and zero shear stress conditions reveal that the constants $a_{\alpha}$ are real and that $a_{1}=a_{2}=0$. Along the solid-fluid interface, the linearization of Laplace equations (4), together with $\hat{\mathbf{n}}=-\mathrm{i} \xi \mathbf{e}_{x}, \hat{\mathbf{t}}=\mathrm{i} \xi \mathbf{e}_{y}$ and $2 \hat{H}=-\xi^{2}$ (according to simple surface geometry results; Aris, 1962), provides two conditions for $a_{3}$ and $a_{0}$ which, once inverted, lead to

$$
\left(\begin{array}{l}
a_{0} \\
a_{3}
\end{array}\right)=\left[\begin{array}{cc}
S+\xi H C & H S \\
-\xi S & -C
\end{array}\right]\left(\begin{array}{c}
1 \\
p_{\mathrm{f}}+\sigma_{0}
\end{array}\right) \times \frac{1}{C S+\xi H} .
$$

The letters $C$ and $S$ in Eq. (C.4) stand for $\cosh (\xi H)$ and $\sinh (\xi H)$, respectively. The last boundary condition to be inspected is along $\mathrm{A}^{\prime} \mathrm{B}^{\prime}$ and depends on whether force or displacement is prescribed on that surface. If the normal displacement is prescribed, its perturbation is zero and the wavenumber $\xi$ has to be equal to $k \pi / L$ ( with $k \in \mathbb{N}$ ) while the no-shear stress conditions is then satisfied by the previous findings on the constants. For force control, the total force on the boundary remains constant constraining the perturbation in stress by

$$
\int_{0}^{H} \hat{\sigma}_{x x}(y) \mathrm{d} y+\left(\sigma_{0}+p_{\mathrm{f}}\right)=0 .
$$

This condition leads to the same equation as the second equation obtained from the linearized Laplace conditions. The second condition for force control is a no-shear stress conditions which is found to be satisfied for the same reason mentioned above if $\xi / \pi L$ is set to any positive, non-zero integer $k$.

Further calculations based on the linearization of the kinetic law (11), which are not discussed here for sake of brevity, leads to the following expression for the stability exponent:

$$
\lambda_{\xi}=-\xi^{2}\left(1-\frac{\left(\sigma_{0}+p_{\mathrm{f}}\right)}{2 G} G_{1}(\xi H)\right)+\xi \frac{\left(\sigma_{0}+p_{\mathrm{f}}\right)^{2}(1-v)}{G} \frac{C^{2}}{C S+\xi H}
$$

with

$$
G_{1}(\xi H)=\frac{\xi H-(1-2 v) C S}{C S+\xi H},
$$

which is found to be real. The function $G_{1}$ of $\xi H$ (a multiple of $H \pi / L$ ) takes values between $1 / 2$ and 1 . Consequently, the factor multiplying $\xi^{2}$ in Eq. (C.6) should be set to first order to 1 to be consistent with the small-strain hypothesis. This simplification leads to expression (24) in the main text for the stability exponent.

This expression (24) for the stability exponent is commented in the main text and we only focus in the rest of this appendix on the limit taken by $\lambda_{\xi}$ as $\xi$ tends to zero. This limit has to be considered for infinitely long rectangular block since $\xi$ remains equal to $k \pi / L$ to satisfy the boundary conditions postulated for the linear stability analysis. Taking this limit in Eq. (C.6), one finds

$$
\lim _{\xi \rightarrow 0} \lambda_{\xi}=\frac{\left(\sigma_{0}+p_{\mathrm{f}}\right)^{2}(1-v)}{G} \frac{1}{2 H} .
$$

This limit is positive or zero and is independent of the type of boundary condition adopted along $\mathrm{A}^{\prime} \mathrm{B}^{\prime}$. This is of course in contradiction with the analysis of the fundamental solution commented with the support of Fig. 5. There, it is seen that the 
fundamental solution is neutrally stable for displacement control and always unstable for force control. Furthermore, a rapid calculation on the fundamental solution for force control (Ghoussoub, 2000) reveals that the stability exponent for a homothetic perturbation, a perturbation in $H$ which preserves the rectangular shape of the block, is identical to the limit found in Eq. (C.7). This discrepancy for displacement control is clarified by looking again at the stability analysis for wavy perturbations noting first that the stress perturbation should be zero for a displacement control test and a perturbation of infinitely long wavelength. The constants $a_{0}$ and $a_{3}$ should then be at least of order 1 in $\xi$. However, it turns out that the system of equations for $\left(a_{0}, a_{3}\right)$ obtained from Laplace conditions is singular for a zero $\xi$. Its solution for $a_{3}$ presented in Eq. (C.4) has a leading term in $(\xi H)^{-1}$ which governs in the limit of zero $\xi H$. It is this singular behavior which results in the difference between the stability exponent in the long-wavelength limit and for homothetic change in thickness.

The structure of the stability analysis just presented is common to many bifurcation and stability problems. We have seen here that a singular limit could explain a discrepancy between homothetic and long-wavelength mode. It is worth noting that a similar discrepancy was found by Leroy and Molinari (1993) for the same type of limit in a 2D shear band analysis. The present simple calculation could provide a first insight on the origin of their results.

\section{References}

Alexander, J.I.D., Johnson, W.C., 1985. Thermomechanical equilibrium in solid-fluid systems with curved interfaces. J. Appl. Phys. 58, 816-824.

Aris, R., 1962. Vectors, Tensors and the Basic Equations of Fluid Mechanics. Prentice-Hall Inc., NJ, Dover, New York, 1989.

Bathurst, R.C.G., 1958. Diagenetic fabrics in some British Dimantian limestones. Liverpool and Manchester Geology 2, 11-36.

Brackbill, J.U., Saltzman, J.S., 1982. Adaptive zoning for singular problems in two dimensions. J. Comput. Phys. 46, 342-368.

den Brok, S.W.J., Morel, J., 2000. The effect of elastic strain on the microstructure of free surfaces of stressed minerals in contact with an aqueous solution. Geophys. Res. Lett. 28, 603-606.

Bowen, R.M., 1967. Toward a thermodynamics and mechanics of mixtures. Arch. Rat. Mech. Anal. 24, 370-403.

Bowen, R.M., Wiese, J.C., 1969. Diffusion in mixtures of elastic materials. Int. J. Engng. Sci. 7, 689-722.

Chiu, C.H., Gao, H., 1993. Stress singularities along a cycloid rough surface. Int. J. Solids Struct. 30, 2981-3012.

Christodoulou, K.N., Scriven, L.E., 1992. Discretization of free surface flows and other moving boundary problems. J. Comput. Phys. 99, 39-55.

Chuang, T.J., Kagawa, K.I., Rice, J.R., Sills, L.B., 1979. Non-equilibrium models for diffusive cavitation of grain interfaces. Acta Metall. 27, 265-284.

Cox, S.F., Paterson, M.S., 1991. Experimental dissolution-precipitation creep in quartz aggregates at high temperature. Geophys. Res. Lett. 18, 1401-1404.

Ghoussoub, J., 2000. Solid-fluid phase transformation within grain boundaries during compaction by pressure solution. Doctoral Thesis, École Nationale des Ponts \& Chaussées-École Polytechnique, Palaiseau, France.

Gibbs, J.W., 1878. On the equilibrium of heterogenous substances. Trans. Conn. Acad. 3, 343-524. Reprinted in The Scientific Papers of J. Willard Gibbs, Vol. 1, Longmans, Green, and Co., Toronto, 1906 (Dover, New York, 1961). 
Gratier, J.P., Guiguet, R., 1986. Experimental pressure solution-deposition on quartz grains: the crucial effect of the nature of the fluid. J. Struct. Geol. 8, 845-856.

Grinfeld, M., 1991. Thermodynamics Methods in the Theory of Heterogeneous Systems. Longman Green and Co., Toronto.

Heidug, W.K., 1991. A thermodynamic analysis of the conditions of equilibrium at nonhydrostatically stressed and curved phase boundaries. J. Geophys. Res. 96 B1, 21,909-21,921.

Heidug, W.K., Lehner, F.K., 1985. Thermodynamics of coherent phase transformation in nonhydrostatically stressed solids. Pure Appl. Geophys. 123, 91-98.

Heidug, W.K., Leroy, Y.M., 1994. Geometrical evolution of stressed and curved solid-fluid phase boundaries. Part 1-transformation kinetics. J. Geophys. Res. 99 B1, 505-515.

Hickman, S.H., Evans, B., 1995. Kinetics of pressure solution at halite-silica interfaces and intergranular films. J. Geophys. Res. B7 (100), 13,113-13, 132.

Hughes, T.J.R., 1987. The Finite Element Method. Prentice-Hall Inc., Englewood Cliffs, NJ.

Kreis, R.I., Thames, F.C., Hassan, H.A., 1986. Application of variational method for generating adaptive grids. AIAA J. 24, 404-410.

Lehner, F.K., Bataille, J., 1984/85. Non-equilibrium thermodynamics of pressure solution. PAGEOPH 122, 53-85.

Leroy, Y.M., Heidug, W.K., 1994. Geometrical evolution of stressed and curved solid-fluid phase boundaries. Part 2-Stability of cylindrical pores. J. Geophys. Res. 99 B1, 517-530.

Leroy, Y.M., Molinari, A., 1993. Spatial patterns and size effects in shear zones: a hyperelastic model with higher-order gradients. J. Mech. Phys. Solids 41, 631-664.

Mullins, W.W., Sekerka, R.F., 1963. Morphological stability of a particle growing by diffusion of heat flow. J. Appl. Phys. 34, 323-329.

Paterson, M.S., 1995. A theory for granular flow accommodated by material transfer via intergranular fluid. In: Spiers, C.J., Takeshita, T. (Eds.), Influence of Fluids on Deformation Processes in Rocks, Tectonophysics, Vol. 245, Elsevier, pp. 135-151.

Raj, R., 1982. Creep in polycrystalline aggregates by matter transport through a liquid phase. J. Geophys. Res. 87, 4731-4739.

Rutter, E.H., 1976. The kinetics of rock deformation by pressure solution. Philos. Trans. R. Soc. Lond. 140, $725-740$.

Rutter, E.H., 1983. Pressure solution in nature, theory and experiment. J. Geol. Soc. Lond. 140, 725-740.

Rimstidt, J.D., Barnes, H.L., 1980. The kinetics of silica-water reactions. Geochim. Cosmochim. Acta 44, 1683-1699.

Schutjens, P.M.T.M., Spiers, C.J., 1999. Intergranular pressure solution in $\mathrm{NaCl}$ : grain-to-grain contact experiments under the optical microscope. Oil Gas Sci. Technol.-Rev. IFP 54, 729-750.

Sleep, N.H., 1995. Ductile creep, compaction and rate and state dependent friction within major faults. Nature 359, 687-692.

Spiers, C.J., Schutjens, P.M.T.M., 1990. Densification of crystalline aggregates by fluid-phase diffusional creep. In: Barber, D.J., Meredith, P.G. (Eds.), Deformation Processes in Minerals, Ceramics and Rocks. Unwin Hyman, London, pp. 334-353.

Spiers, C.J., Schutjens, P.M.T.M., Brzesowsky, R.H., Peach, C.J., Liezenberg, J.L., Zwart, H.J., 1990. Experimental determination of constitutive parameters governing creep of rock salt by pressure solution. In: Knipe, R., Rutter, E.H. (Eds.), Deformation Mechanisms, Rheology and Tectonics. Geol. Soc. Lond. Spec. Pub. 54, 215-353.

Srolovitz, D.J., 1989. On the stability of surfaces of stressed solids. Acta Metall. 37, 621-625.

Tsiveriotis, K., Brown, R.A., 1992. Boundary-conforming mapping applied to computations of highly deformed solidification interfaces. Int. J. Numer. Meth. Fluids 14, 981-1003.

Truesdell, C., 1977. A First Course in Rational Continuum Mechanics. Academic Press, New York.

Truskinovsky, L.M., 1984. The chemical-potential tensor. Geochem. Int. 21, 22-36.

Urai, J.L., Spiers, C.J., Zwart, H.J., Lister, G.S., 1986. Weakening of rocksalt by water during long-term creep. Nature 324, 554-557.

Yang, W.H., Srolovitz, D.J., 1994. Surface morphology evolution in stressed solids: surface diffusion controlled crack initiation. J. Mech. Phys. Solids 42, 1551-1574. 
Wang, W., Suo, Z., 1997. Shape change of a pore in a stressed solid via surface diffusion motivated by surface and elastic energy variation. J. Mech. Phys. Solids 45, 709-729.

Welton, J.E., 1984. SEM Petrology Atlas. Methods in Exploration Series. AAPG, Tulsa, USA.

Weyl, P.K., 1959. Pressure solution and force for crystallization - a phenomenological theory. J. Geophys. Res. 64, 2001-2025.

Zabaras, N., Yimin, Y., Richmond, O., 1991. On the calculation of deformations and stresses during axially symmetric solidification. J. Appl. Mech. 58, 865-871. 